\title{
RIESGOS SISTÉMICOS EN EL MERCADO INTERBANCARIO EN VENEZUELA, 2004-2014*
}

\author{
Recibido: 01 de junio de 2016 • Aprobado: 23 de enero de 2017 \\ DOI: $10.22395 /$ seec.v20n42a4

$$
\begin{gathered}
\text { Carolina Pagliacci** } \\
\text { Jennifer Peña*** }
\end{gathered}
$$

\section{RESUMEN}

Este trabajo muestra la aplicación del modelo núcleo-periferia para medir dos dimensiones del riesgo sistémico en el mercado interbancario venezolano: conectividad y patrones de fondeo entre bancos. El período de estudio es de interés porque contiene un episodio de ajuste financiero en el año 2009. Los resultados muestran que posterior a esta fecha, la conectividad del mercado parece haberse reducido, lo que podría redundar en menor riesgo sistémico de contagio. En contraposición, los patrones de fondeo parecen haberse modificado y sugieren que los bancos con más permanencia en el núcleo tienden a tener necesidades de liquidez crecientes, apuntando a mayor riesgo de iliquidez en dichos bancos. Asimismo, la aplicación del modelo contribuye a identificar bancos donde debe focalizarse la supervisión.

\section{PALABRAS CLAVE}

Sistema financiero; modelo núcleo-periferia; mercado interbancario; riesgo sistémico; Venezuela.

\section{CLASIFICACIÓN JEL}

D85, G21, L14

\section{CONTENIDO}

Introducción; 1. Modelo núcleo-periferia; 2. Aplicación al sistema bancario venezolano; 3. Análisis de resultados; 4. Conclusiones; Bibliografía.

* Este artículo es parte del trabajo de investigación realizado dentro de la Oficina de Investigaciones Económicas del Banco Central de Venezuela para estudiar la estructura del mercado interbancario venezolano durante el período 2004-2014. Las opiniones emitidas en este artículo son responsabilidad de los autores y no comprometen la visión que el Banco Central de Venezuela pueda tener sobre el tema.

** Economista, Universidad Católica Andrés Bello, Caracas, Venezuela. PhD en Economía, Texas A\&M University, Texas, Estados Unidos. Investigadora senior en el Banco Central de Venezuela, Caracas, Venezuela. Dirección Postal: 1010, Esquina Las Carmelitas, Edificio Sede, piso 2, Oficina de Investigaciones Económicas, Caracas, Venezuela. Teléfono +58212 8015919. Correo electrónico: cpagliac@bcv.org.ve

*** Matemática, Universidad Central de Venezuela, Caracas, Venezuela. Magíster en Matemática, Universidad Central de Venezuela, Caracas, Venezuela. Analista Económica en el Banco Central de Venezuela, Caracas, Venezuela. Dirección Postal: 1010, Esquina Las Carmelitas, Edificio Sede, piso 2, Oficina de Investigaciones Económicas, Caracas, Venezuela. Teléfono +58212 5367252. Correo electrónico: jenpena@bcv.org.v 


\section{SYSTEMIC RISK IN THE VENEZUELAN INTERBANK MARKET, 2004-2014 ABSTRACT}

This paper presents a core-periphery model application for measuring two systemic risk dimensions in the Venezuelan inter-bank market: connectivity and funding patterns between banks. The period of study is of special interest given that it includes a financial adjustment during 2009. Results evidence that after this date, market connectivity seems to have dropped, which could lead to a smaller systemic risk spread. In the other hand, funding patterns seem to have been modified and suggest that banks, which belong to the core, tend to present increasing liquidity needs, which increases the liquidity risks of this banks. Likewise, the model application helps to identify which banks should be more closely supervised.

\section{KEY WORDS}

Financial systems; core-periphery model; interbank market; systemic risk; Venezuela.

\section{JEL CLASSIFICATION}

D85, G21, L14

\section{CONTENT}

Introduction; 1. Core-periphery model; 2. Application to the Venezuelan banking system; 3. Result analysis; 4. Conclusions; Bibliography.

\section{RISCOS SISTÉMICOS NO MERCADO INTERBANCÁRIO NA VENEZUELA, 2004 - 2014}

\section{RESUMO}

Este trabalho mostra a aplicação do modelo núcleo-periferia para medir duas dimensões do risco sistémico no mercado interbancário venezuelano: conectividade e padrões de ancoragem entre bancos. O período de estudo é de interesse porque contém um episódio de ajuste financeiro no ano 2009. Os resultados mostram que posterior a esta data, a conectividade do mercado parece ter reduzido, o que poderia redundar em menor risco sistémico de contágio. Em contraposição, os padrões de ancoragem parecem ter sido modificado e sugerem que os bancos com mais permanência no núcleo tendem a ter necessidades de liquidez crescentes, apontando a maior risco de iliquidez em ditos bancos. Assim mesmo, a aplicação do modelo contribui a identificar bancos onde deve focalizar-se a supervisão.

\section{PALAVRAS CHAVE}

Sistema financeiro; modelo núcleo-periferia; mercado interbancário; risco sistémico; Venezuela.

\section{CLASSIFICAÇÃO JEL}

D85, G21, L14

\section{CONTEÚDO}

Introdução; 1. Modelo núcleo-periferia; 2. Aplicação ao sistema bancário venezuelano; 3. Análise de resultados; 4. Conclusões; Bibliografia. 


\section{INTRODUCCIÓN}

Las autoridades responsables de velar por la estabilidad financiera están interesadas en monitorizar la actividad de los bancos en el mercado interbancario para detectar potenciales eventos con efectos sistémicos. Siguiendo a Frait y Komárková (2011) los riesgos sistémicos tienen dos aspectos sobre los cuales se basa su regulación: temporal y transversal. El primero analiza el comportamiento de los riesgos en el tiempo, mientras que el segundo evalúa la existencia, distribución y correlación de riesgos entre instituciones en un momento dado. La dimensión temporal monitoriza indicadores de precios de los activos, agregados monetarios, entre otros, mientras que la transversal estudia la interconexión entre entidades, y estructura del sistema financiero, así como los eventos de estrés que pueden ser transmitidos entre bancos o contagios. Esta segunda dimensión se ha apoyado en la teoría de redes, la cual contribuye a analizar y entender las conexiones que se forman en el sistema financiero debido a la transferencia de activos entre entidades. El riesgo de contagio o riesgo de red se atribuye a una institución financiera cuando esta se encuentra demasiado interconectada para caer (too interconnected to fail), (al respecto ver Komárkova, Hausenblas y Frait, 2012). En general, las conexiones y la estructura de una red financiera pueden influir en la magnitud del riesgo sistémico, tal como lo refieren Allen y Gale (2000), Fricke y Lux (2012), Anand, Craig y Von Peter (2014), Liu y Quiet (2015) y Craig, Fecht y Tümer-Alkan (2015)1.

En este contexto, el objetivo general del trabajo es utilizar la estructura de redes para evaluar el aspecto transversal del riesgo sistémico que pueda originarse en el mercado interbancario. Por ejemplo, identificar si el mercado interbancario tiene alguna estructura particular puede contribuir con el objetivo de las autoridades encargadas de velar por la estabilidad del sistema, tal como señalan Yellen (2013), Tirole (2011), Upper (2011), entre otros. Asimismo, identificar los bancos que más se relacionan con sus pares puede ayudar a comprender en qué bancos la ocurrencia de problemas de solvencia puede transmitirse más rápido al resto de los bancos, tal como lo señalan, Aldasoro y Alves (2015). Por otro lado, determinar si los bancos más conectados proveen de liquidez al sistema o son los demandantes netos de recursos permite evaluar en qué medida la ocurrencia de episodios de iliquidez con mayor impacto sistémico es más probable.

En este sentido, el objetivo más específico del trabajo es utilizar el modelo estructural de redes denominado "núcleo-periferia" para entender dos dimensiones

El estudio de las conexiones se ha convertido en un elemento clave en los estudios del riesgo sistémico del sector bancario. Hay estudios de este tipo desarrollados por el Fondo Monetario Internacional (IMF, por sus siglas en inglés), el Banco de Pagos Internacionales (BIS, por sus siglas en inglés) y Consejo de Estabilidad Financiera (FSB, por sus siglas en inglés). Al respecto ver FSB, IMF y BIS (2009). 
concretas del riesgo interbancario: la conectividad y los patrones de fondeo de los bancos. En tanto, la conectividad intenta capturar el grado de contagio que de un evento de insolvencia o iliquidez, los patrones de fondeo pretenden detectar sobre qué bancos o grupo de bancos existe un mayor riesgo de iliquidez. Este análisis incorpora el aspecto temporal del riesgo al evaluar el cambio en la conectividad y en los patrones de fondeo en el tiempo.

El modelo núcleo-periferia provee una clasificación jerárquica de los bancos (en núcleo o periferia), donde el núcleo representa a los bancos más conectados. La aplicación de este modelo al mercado interbancario es propuesta por Craig y von Peter (2014). Según esta visión, los bancos ubicados en el núcleo se prestan recursos entre sí, e intermedian con otros bancos que no se extienden crédito entre ellos, llamados periferia. Esta representación de las características generales y de forma compacta de la red interbancaria, en cierta forma, facilita la supervisión al identificar los bancos más conectados. En estudios empíricos aplicados a otros países, como por ejemplo, Craig y von Peter (2014) en Alemania, in 't Veld y van Lelyveld (2014) en Países Bajos, Solis-Montes (2013) en México, Fricke y Lux (2012) en Italia y Langfield, Liu y Ota (2014) en Reino Unido, se ha encontrado que los bancos del núcleo tienden a ser los más grandes y que, en muchos casos participan en el sistema como mayoristas del mercado monetario (market makers).

A partir de la clasificación jerárquica que proporciona el modelo, también se evalúan los patrones de financiamiento de los bancos, a partir de indicadores calculados por bloques (núcleo versus periferia) e indicadores aplicados a cada banco del sistema. El grado de conectividad de un banco en particular se asocia con su frecuencia de permanencia en el núcleo. El patrón de financiamiento se obtiene calculando la diferencia entre los fondos prestados y los demandados durante dicha permanencia en el núcleo. Estos indicadores por banco no han sido calculados en trabajos previos que aplican el modelo núcleo-periferia, los cuales tienden a centrarse solamente en la conectividad entre los grupos núcleo y periferia. En este sentido, nuestro trabajo provee una extensión del uso de este tipo de modelos para mejorar la evaluación del riesgo en el mercado interbancario.

Para la estimación del modelo núcleo-periferia se utiliza el algoritmo aplicado en Craig y von Peter (2014), el cual clasifica de forma óptima los bancos del mercado interbancario en núcleo o periferia. El proceso de optimización consiste en encontrar aquella partición de bancos que minimiza los errores que se producen entre las conexiones realmente mantenidas por los bancos y las conexiones que se derivan de la estructura teórica entre el núcleo y la periferia. Las conexiones se refieren a las que surgen entre los bancos que se prestan recursos en el mercado interbancario 
venezolano durante el lapso de un mes. Los datos de los préstamos interbancarios corresponden a las transacciones realizadas diariamente por todos los bancos del sistema, las cuales son obtenidas directamente de los registros mantenidos en el Banco Central de Venezuela. Este trabajo, a diferencia de los de Furfine (2003), Upper y Worms (2004), Boss et al. (2004), Degryse y Nguyen (2007), y Anand, Craig y von Peter (2014), no requiere hacer una estimación previa de la matriz de conexiones entre bancos, pues esta es completamente observada. Otros trabajos que utilizan información observada de transacciones son Bastos e Santos y Cont (2010), Fricke y Lux (2012), Solis-Montes (2013) y Craig y von Peter (2014).

La aplicación del modelo al caso venezolano se efectúa para el período 20042014, el cual tiene dos elementos por demás interesantes. Por un lado, analiza un período de estudio durante el cual está en vigencia un control cambiarioº ${ }^{2}$ En este sentido, la evidencia obtenida podría atribuirse parcialmente al particular entorno macroeconómico, el cual presenta características muy diferentes al de países con mercados cambiarios no-regulados. Por otro lado, durante este período también se llevó a cabo una serie de fusiones e intervenciones, entre 2009 y 2010, que podrían haber afectado de forma importante la estructura del mercado 3 . Desde el punto de vista estadístico, considerar el análisis a partir del 2004 nos permite capturar eventos importantes del sistema y, a la vez, trabajar con información asociada a un mismo régimen cambiario.

Entre los resultados más importantes se encuentra que, a diferencia de los estudios empíricos de otros países, el núcleo en nuestro sistema está conformado mayormente por bancos relativamente pequeños que, además, son demandantes netos de recursos. En la periferia encontramos los bancos que proveen de liquidez al núcleo. Esto, en cierta forma, podría atribuirse a la existencia de un control cambiario que hace que la intermediación interbancaria se reduzca, y

2 A inicios de 2003 el Ejecutivo Nacional y el Banco Central de Venezuela adoptaron un régimen de administración de divisas (control de cambios), donde las transacciones de la balanza comercial se canalizan a un tipo de cambio pre-establecido y las transacciones de capital pueden ser financiadas a un tipo de cambio paralelo o no oficial. En términos generales se puede entender el establecimiento del control de cambio como la aparición de mercados de cambio duales, en el que el precio no-oficial de la moneda presenta una prima importante respecto al precio oficial.

3 A final del año 2009 y durante el 2010, la Superintendencia de bancos intervino dieciocho entidades bancarias. La medida fue dictada por incumplimiento de normas administrativas, entre las cuales se encontraba el aumento de capital sin especificar el origen de los fondos, los índices patrimoniales, negociaciones fraudulentas con el dinero de los ahorristas, escasez de liquidez para asumir responsabilidades e incumplimiento recurrente de las carteras dirigidas. La idea de las intervenciones era fortalecer la actividad financiera del Estado, reforzar las instituciones que prestan servicios de banca universal, reagrupar a las llamadas instituciones financieras especializadas, así como brindar a la mayor parte de la población venezolana los servicios que prestan los organismos financieros, Cáribas (2011). 
prevalezcan solo aquellas relaciones basadas en requerimientos de liquidez. Otro resultado importante se refiere a los cambios en la conectividad y en el patrón de fondeo de los bancos que se registra a partir de las fusiones e intervenciones bancarias.

Los cambios observados a partir de las fusiones e intervenciones financieras son fundamentalmente dos. Primero, la conectividad del mercado parece haberse reducido de forma sustancial, debido a la reducción que se observa en el tamaño y las conexiones del núcleo. Esta menor conectividad podría hacer pensar en una disminución del riesgo sistémico, en tanto la ocurrencia de choques idiosincráticos a bancos tendría una menor probabilidad de ser transmitido al resto de los bancos. En otras palabras, al existir menos bancos muy conectados (los del núcleo), los eventos de insolvencia en bancos de la periferia podrían causar menores repercusiones sistémicas. En segundo lugar, los bancos del núcleo redujeron sus vínculos y volúmenes transados con el resto de los bancos del núcleo, pero, a su vez, incrementaron sus vínculos y volúmenes transados con los bancos de la periferia. Sin embargo, estas mayores conexiones del núcleo en relación con la periferia ocurren principalmente para obtener recursos de esta. Esto hace presumir que hay una mayor probabilidad de iliquidez para estos bancos que permanecen en el núcleo, generando un mayor riesgo sistémico.

Este artículo se encuentra estructurado en cuatro secciones. En la primera sección se presenta el modelo núcleo-periferia. En la segunda, se describe la aplicación al caso venezolano: los datos utilizados y la información estadística que da cuenta del ajuste del modelo. Luego, se presentan los resultados e indicadores relevantes para analizar la conectividad y los patrones de fondeo por grupos de bancos y bancos individuales. En la cuarta sección se presentan las conclusiones.

\section{MODELO NÚCLEO-PERIFERIA}

En esta sección introducimos la estructura teórica del modelo núcleo-periferia. De manera general, una red está formada por nodos que representan agentes, (países, bancos, entre otros), y los vínculos entre ellos representan las relaciones en términos de exposición o flujos intercambiados, entre otros. Las relaciones pueden representarse de dos formas: binaria y ponderada. En la forma binaria se indica con 1 o 0 la presencia o ausencia de exposición entre nodos, respectivamente, mientras que en la ponderada se incorpora el tamaño de la relación con la magnitud del vínculo entre nodos. El análisis de redes es un conjunto de métodos que describe la posición de los nodos en la red y evalúa la estructura que forma la red en su conjunto, tal como lo refiere Minoiu (2012). 
Borgatti y Everett (1999) fueron los primeros en dar la definición formal de la estructura núcleo-periferia, a pesar de haber sido una noción implementada en diferentes campos y sistemas complejos ${ }^{4}$. Estos autores definen la estructura núcleo-periferia basados en las bondades conceptuales de la teoría de redes, a partir de un núcleo conectado a sí mismo, que en términos matriciales correspondería a una matriz de l's, mientras que los elementos de la periferia no mantienen conexión entre ellos, es decir, una matriz de 0's. Como bien lo resumen Langfield, Liu y Ota (2014) en el tabla 1.

Tabla 1. Estructura núcleo-periferia

\begin{tabular}{|l|l|l|}
\hline & \multicolumn{1}{|c|}{ Núcleo (C) } & \multicolumn{1}{c|}{ Periferia (P) } \\
\hline Núcleo $(\mathrm{C})$ & Sub-matriz de 1's (CC) & Depende del caso de estudio (CP) \\
\hline Periferia (P) & Depende del caso de estudio (PC) & Sub-matriz de 0's (PP) \\
\hline
\end{tabular}

Fuente: adaptación de Langfield, Liu y Ota (2014).

En la formulación matricial de la estructura estilizada, los elementos en los bloques fuera de la diagonal (núcleo-periferia y periferia-núcleo) pueden tomar cualquier valor según el fenómeno estudiado. Borgatti y Everett (1999) se centran en las sub-matrices de l's y 0's ubicadas en la diagonal'.

En el caso de las redes financieras, y en particular de las redes interbancarias, su aplicación la desarrollan Craig y von Peter (2014), quienes suponen que los bancos del núcleo deberían prestar y pedir recursos a, al menos, un banco de la periferia. Ellos consideran que los bancos del núcleo deberían desempeñar un rol de intermediación, pero no limitado entre los miembros del núcleo. Se define como intermediario en el sistema interbancario a un banco que actúa simultáneamente como prestamista y prestatario. Los bancos del núcleo les prestan a los de su mismo nivel e intermedian entre otros bancos, llamados periferia que solo participan en el mercado interbancario a través de los bancos del núcleo. Esta estructura jerárquica puede ser expresada a partir de las relaciones presentes entre los bancos núcleo y periferia, como bien lo señalan Craig y von Peter (2014) en las siguientes condiciones:

I. Los bancos del núcleo se prestan recursos entre sí

II. Los bancos de la periferia no les prestan a otros de la periferia

III. Los bancos del núcleo les prestan a, al menos, un banco de la periferia

4 En adelante consideraremos la notación del núcleo como C, por ser en inglés Core.

5 Información adicional sobre la estructura núcleo-periferia la podemos encontrar en Hojman y Szeidl (2008). 
IV. Los bancos del núcleo les piden recursos a, al menos, un banco de la periferia.

A partir de las condiciones anteriores, se generan relaciones entre los niveles núcleo y periferia, basadas en la relación bilateral y caracterizadas por la intermediación entre los dos niveles (condiciones III y IV).

La definición dada puede tener una representación estructural basada en las definiciones utilizadas en redes ${ }^{6}$. Al considerar cada nodo como un banco, las posiciones interbancarias entre ellos constituyen una red, que puede ser representada como una matriz cuadrada de dimensión nxn con nigual al número de bancos en el sistema. Un elemento $(\mathrm{i}, \mathrm{j})$ de esta matriz representa el valor del crédito que el banco $i$ le dio al banco j. La fila i muestra la exposición interbancaria bilateral del banco $i$ con cada uno de los otros bancos, mientras que, la columna $j$ muestra los pasivos interbancarios del mismo banco con cada uno de los otros bancos en el sistema. La diagonal $(i, i)$ es cero al considerar los bancos como una sola entidad que no se presta a sí misma.

Ahora bien, en la definición del modelo núcleo-periferia, no consideraremos la matriz de valores sino la de vínculos, los cuales indican la presencia de conexiones con 1 y ausencia de conexiones con 0 . Llamaremos $\mathrm{N}$ la matriz del sistema interbancario observada de tamaño $n \times n$. Es importante notar que la matriz tiene vínculos dirigidos, es decir, $\mathrm{N}_{i j} \neq \mathrm{N}_{j i}$, donde $\mathrm{N}_{i j}$ es la entrada $(i, j)$ de la matriz $\mathrm{N}$.

Por otro lado, las relaciones I-VI pueden ser mapeadas en una matriz binaria (ecuación [1]), M, formada por bloques o sub-matrices, a partir de la naturaleza de los vínculos de los bancos tipo núcleo (C) y periferia (P):

$$
M=\left(\begin{array}{ll}
C C & C P \\
P C & P P
\end{array}\right)
$$

El bloque denotado por CC (núcleo-núcleo) resume cómo los bancos del núcleo se relacionan entre sí (condición I), por lo que CC es una sub-matriz de unos (con ceros en la diagonal). De la misma manera, como los bancos de la periferia no se prestan recursos entre sí, PP (periferia-periferia) es una sub-matriz de ceros, (condición II). Ahora bien, CP (núcleo-periferia) recoge los vínculos correspondientes a los bancos del núcleo que le prestan a la periferia, así que este bloque debe ser fila regular, es decir, contiene al menos un vínculo en cada fila (condición III); para más detalles se puede consultar

$\overline{6}$ Información adicional sobre redes la podemos encontrar en Soramäki et al. (2007). 
Doreian, Batagelj y Ferligoj (2005). De igual manera, como los bancos núcleo le piden prestado a, al menos, un banco de la periferia, PC (periferia-núcleo) es una sub-matriz columna regular, es decir, con al menos un uno en cada columna (condición IV).

El tamaño de los bloques será determinado por el número de bancos en cada nivel. Si $c$ bancos se encuentran en el núcleo, y la red está formada por $n$ bancos, entonces la sub-matriz $\mathrm{CP}$, tendrá dimensión $c x(n-c)$ y será de tamaño $(n-c) x c$.

Como vemos, $\mathrm{M}$ es un modelo núcleo-periferia perfecto o ideal que contiene cómo debería comportarse cada bloque (CC, CP, PP, PC) y sirve como benchmark para cualquier red, $\mathrm{N}$, observada en el mercado interbancario.

El ajuste que se realiza involucra dos pasos como bien lo señalan Craig y von Peter (2014): primero, se define una medida de distancia entre la red observada, N, y el modelo M asociado de la misma dimensión. Luego, se resuelve el problema de encontrar la partición óptima (la que minimice la distancia entre $\mathrm{M} \mathrm{y} \mathrm{N}$ ) de la red en núcleo y periferia.

La medida de distancia utilizada es un error total (ecuación [3]) que agrega el

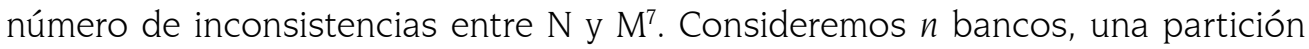
arbitraria de $c$. bancos en el núcleo y $n-c$ en la periferia. Denotemos el conjunto ordenado de bancos en el núcleo por $C=\{1,2,3, \ldots . c\}$ con la correspondiente permutación de N. La ecuación [2] contiene los errores en cada bloque:

$$
E=\left(\begin{array}{cc}
c(c-1)-\sum_{i \in C} \sum_{j \in C} N_{i j} & (n-c) \sum_{i \in C} \max \left\{0,1-\sum_{j \notin C} N_{i j}\right\} \\
(n-c) \sum_{j \in C} \max \left\{0,1-\sum_{j \notin C} N_{i j}\right\} & \sum_{i \notin C} \sum_{j \notin C} N_{i j}
\end{array}\right)
$$

El error total agrega los errores en las cuatro sub-matrices:

$$
\mathrm{E}_{11}=c(c-1)-\sum_{i \in \mathrm{C}} \sum_{j \in \mathrm{C}} \mathrm{N}_{i j}
$$

$7 \quad$ Es una generalización del modelo blockmodeling de Doreian y Bataglj y Ferligoj (2005) 


$$
\begin{gathered}
\mathrm{E}_{12}=(n-c) \sum_{j \in C} \max \left\{0,1-\sum_{j \notin C} \mathrm{~N}_{i j}\right\} \\
\mathrm{E}_{21}=(n-c) \sum_{i \in \mathrm{C}} \max \left\{0,1-\sum_{j \notin C} \mathrm{~N}_{i j}\right\} \\
\mathrm{E}_{22}=\sum_{i \notin \mathrm{C}} \sum_{j \notin \mathrm{C}} \mathrm{N}_{i j}
\end{gathered}
$$

Donde E es la matriz de errores de dimensión 2x2; $E_{11}$ (ecuación [4]) son los errores en CC o vínculos faltantes; $E_{22}$ (ecuación [7]) son los errores en PP o vínculos que no deberían existir; $\mathrm{E}_{12}$ (ecuación [5]) errores en CP o inconsistencias en la condición de ser fila regular y $E_{21}$ (ecuación [6]) son los errores en PC o en la condición de ser columna regular.

El núcleo óptimo, $C^{*}$, es el conjunto de bancos que generan la distancia mínima entre el modelo $\mathrm{M}$ asociado y la correspondiente red observada $\mathrm{N}$ :

$$
\mathrm{C}^{*}=\{\mathrm{C} \in \Gamma / e(\mathrm{C}) \leq e(c) \forall c \in \Gamma\}
$$

Donde'I el conjunto de todos los subconjuntos de $\{1, \ldots, n\}$.

A partir de la definición de distancia dada, la solución que genera la partición óptima satisface propiedades que pueden ser resumidas en la siguiente proposición que presentan Craig y von Peter (2014):

1) La presencia de intermediarios es necesaria y suficiente para detectar una estructura núcleo-periferia en una red observada:

a) Una red sin intermediarios no tiene núcleo.

b) Una red con intermediarios tiene núcleo (y periferia).

2) El núcleo es un subconjunto de los intermediarios:

a) Todos los bancos del núcleo son intermediarios.

b) Los intermediarios no son parte del núcleo sino prestan o piden prestado a la periferia.

La primera propiedad está relacionada con la existencia y la segunda con el núcleo como subconjunto de los intermediarios. Otro elemento que se puede deducir, a partir de la segunda propiedad, es que, en el óptimo las submatrices de la matriz $\mathrm{E}$, de errores que se encuentra fuera de la diagonal tienen error cero, ya que están asociadas a la relación del núcleo con la periferia, lo que implica que el error del núcleo óptimo se simplifique en la ecuación [8]: 


$$
e\left(C^{*}\right)=\frac{E_{11}+E_{22}}{\sum_{i} \sum_{j} N_{i j}}
$$

Es decir, CC y PP son las submatrices que generan errores a través de vínculos faltantes (en CC) y aquellos que no deberían existir (en PP).

Ahora bien, en el análisis de redes la existencia de características similares a una estructura núcleo-periferia tiene implicaciones importantes: tenemos pocos participantes centrales y muchos periféricos; el sistema es robusto ante la extracción aleatoria de participantes (fallas), pero frágil ante la extracción determinística de participantes del núcleo (choque) ya que el sistema se desarticula al suprimir los participantes más conectados, tal como lo señalan León, Pérez y Mariño (2015) y Barabási (2014). Lo anterior sugiere la importancia de los participantes del núcleo en el sistema. Una vez explicados los elementos teóricos del modelo núcleo-periferia, pasaremos a implementarlo en el sistema venezolano.

\section{APLICACIÓN AL SISTEMA BANCARIO VENEZOLANO: DATOS UTILIZADOS Y ESTIMACIÓN DEL MODELO}

Los datos utilizados provienen del Sistema Electrónico de Transferencias (SET), que es un sistema computarizado del Mercado Venezolano de Fondos Overnight que permite comprar o vender fondos interbancarios a cualquier tasa de interés de común acuerdo entre los que pactan una transacción (mercado de órdenes limitadas). Este sistema es similar a los empleados en los mercados de bolsas internacionales, con la diferencia de que permite a los oferentes de fondos asignar líneas de crédito a sus potenciales socios comerciales y requerir colaterales de hasta $100 \%$ de cobertura de forma discrecional, tal como lo señala Pagliacci (2008).

El SET comenzó su funcionamiento en octubre del año 2000; sin embargo, nuestro análisis se realiza desde enero de 2004 a diciembre 2014, ya que, a inicios de 2003, se estableció un régimen de administración de divisas o control de cambio. Con la introducción del control cambiario, las operaciones en el mercado interbancario tendieron a reducirse debido a un aumento generalizado de la cantidad de dinero de la economía. Por tanto, la selección del período analizado busca garantizar un período de operaciones homogéneo, al menos desde el punto de vista de las condiciones macroeconómicas generales. Tal como se reseña a lo largo del trabajo, este período también contiene una serie de fusiones e intervenciones bancarias que

\footnotetext{
8 Las autoras suministrarán a los investigadores interesados los datos binarios utilizados en el estudio para
} construir las matrices de adyacencia, mediante solicitud al correo electrónico: cpagliac@bcv.org.ve 
se dieron en Venezuela a finales de 2009 y en el 2010. En este sentido, esta muestra provee información relevante de cómo la estructura del mercado interbancario cambia con la ocurrencia de estas intervenciones selectivas, y cómo los indicadores derivados del modelo núcleo-periferia capturan estos cambios.

A partir de la información proporcionada por el SET, se construyen las matrices de exposición bilateral en cada mes (en total 132 meses o redes), la cual indica la presencia o ausencia de exposición al crédito en valores monetarios (en bolívares) entre los 56 bancos que participan en el sistema. A partir de esta información, se generan las matrices de adyacencias binarias, $\mathrm{N}_{t}$ (donde $t$ indica el mes), a las que se les aplica el algoritmo propuesto por Craig y von Peter (2014) para obtener el núcleo que genera el mínimo en la función de error total9. Las matrices $\mathrm{N}_{t}$ son de dimensión $56 \times 56$ y sus entradas están formadas por unos y ceros, indicando la presencia o ausencia de exposición al crédito, respectivamente. A partir de estas matrices se estudiará la topología del mercado interbancario considerando únicamente datos reales sin tener que hacer estimaciones previas de las conexiones.

Antes de iniciar el análisis se ilustra con un ejemplo adaptado de Craig y von Peter (2014) el método utilizado para seleccionar el núcleo óptimo. Considere un sistema hipotético formado por ocho bancos: $\mathrm{B}_{i}$, con $i=1,2, \ldots, 8$. En [9] se tiene la matriz de exposición (volumen) asociada al sistema, una matriz cuadrada de dimensión 8x8, donde se colocan los mismos bancos en las filas y en las columnas, para evaluar sus relaciones:

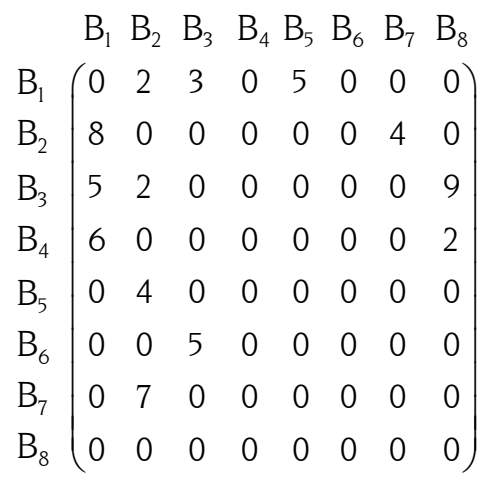

Las filas señalan lo que cada banco les presta a los otros 7 bancos del sistema y en las columnas se representa lo que cada banco les debe a los otros, es decir, en la lectura por filas se tiene a los prestamistas y en las columnas, a los prestatarios.

\footnotetext{
9 El algoritmo de optimización utilizado para encontrar el tamaño óptimo del núcleo fue elaborado por Craig y Von Peter (2014), quienes lo facilitaron para esta investigación.
} 
En el ejemplo, el banco $\mathrm{B}_{3}$ le prestó al banco $\mathrm{B}_{8} 9$ unidades monetarias y le debe al banco $B_{1}$ unas 3 unidades monetarias.

La matriz binaria $(\mathrm{N})$ asociada a este sistema se encuentra en [10]:

\begin{tabular}{|c|c|c|c|c|c|c|c|c|}
\hline & $\mathrm{B}_{1}$ & $\mathrm{~B}_{2}$ & $\mathrm{~B}_{3}$ & & & & & $3_{8}$ \\
\hline $\mathrm{B}_{1}$ & $(0$ & 1 & 1 & 0 & & U & & 0) \\
\hline $\mathrm{B}_{2}$ & 1 & 0 & 0 & 0 & 0 & 0 & & 0 \\
\hline $\mathrm{B}_{3}$ & 1 & 1 & 0 & 0 & 0 & 0 & 0 & 1 \\
\hline $\mathrm{B}_{4}$ & 1 & 0 & 0 & 0 & 0 & 0 & 0 & 1 \\
\hline $\mathrm{B}_{5}$ & 0 & 1 & 0 & 0 & 0 & 0 & 0 & 0 \\
\hline $\mathrm{B}_{6}$ & 0 & 0 & 1 & 0 & 0 & 0 & 0 & 0 \\
\hline $\mathrm{B}_{7}$ & 0 & 1 & 0 & 0 & 0 & 0 & 0 & 0 \\
\hline $\mathrm{B}_{8}$ & 0 & 0 & 0 & 0 & 0 & 0 & 0 & 0 \\
\hline
\end{tabular}

Los bancos $\left\{\mathrm{B}_{4}, \mathrm{~B}_{6}, \mathrm{~B}_{8}\right\}$ prestan o piden recursos, no ambas, por lo que los intermediarios son los bancos restantes: $\left\{B_{1}, B_{2}, B_{3}, B_{5}, B_{7}\right\}$. Por ejemplo, el banco $B_{3}$ intermedia entre $B_{6} y_{8}$, ya que $B_{6}$ le presta a $B_{3}$ y este, a su vez, le presta a $B_{8}$, pero $\mathrm{B}_{6}$ y $\mathrm{B}_{8}$ no intercambian recursos directamente. Ahora bien, si suponiendo que el subconjunto $\left\{\mathrm{B}_{1}, \mathrm{~B}_{2}, \mathrm{~B}_{3}\right\}$ son los candidatos ideales del núcleo, entonces la estructura ideal (M) que satisface las condiciones (I), (III) y (IV) de la definición, se tiene en [11]:

\begin{tabular}{|c|c|c|c|c|c|c|c|c|}
\hline & $\mathrm{B}_{1}$ & $\mathrm{~B}_{2}$ & $\mathrm{~B}_{3}$ & $\mathrm{~B}_{4}$ & $B$ & $\mathrm{~B}_{6}$ & $B_{-}$ & \\
\hline $\mathrm{B}_{1}$ & $(0$ & 1 & 1 & 0 & 1 & 0 & 0 & 0 \\
\hline $\mathrm{B}_{2}$ & 1 & 0 & 1 & 0 & 0 & 0 & 1 & \\
\hline $\mathrm{B}_{3}$ & 1 & 1 & 0 & 0 & 0 & 0 & 0 & \\
\hline $\mathrm{B}_{4}$ & 1 & 0 & 0 & 0 & 0 & 0 & 0 & 0 \\
\hline $\mathrm{B}_{5}$ & 0 & 1 & 0 & 0 & 0 & 0 & 0 & 0 \\
\hline $\mathrm{B}_{6}$ & 0 & 0 & 1 & 0 & 0 & 0 & 0 & 0 \\
\hline $\mathrm{B}_{7}$ & 0 & 1 & 0 & 0 & 0 & 0 & 0 & 0 \\
\hline $\mathrm{B}_{8}$ & 0 & 0 & 0 & 0 & 0 & 0 & 0 & 0 \\
\hline
\end{tabular}

Los bancos $\mathrm{B}_{5}$ y $\mathrm{B}_{7}$ son únicamente intermediarios porque no están conectados correctamente con los otros bancos, de acuerdo con la definición. Cuando se compara la matriz $\mathrm{M}$ con la $\mathrm{N}$, se observa que un banco del núcleo $\left(\mathrm{B}_{2}\right)$ no le presta a otro banco del núcleo $\left(\mathrm{B}_{3}\right)$, y que un banco de la periferia $\left(\mathrm{B}_{4}\right)$ le presta a otro de su mismo nivel $\left(\mathrm{B}_{8}\right)$. Por lo tanto, en la ecuación [2] de los errores $\mathrm{E}_{11}=\mathrm{E}_{11}=1 \mathrm{y}$ $E_{12}=E_{21}=0$, luego, al no encontrar otra partición que minimice este error, se tiene que los tres bancos seleccionados forman el núcleo y $e\left(C^{*}\right)=2 / 13$. 


\subsection{Ajuste del modelo}

En esta sección evaluamos el ajuste estadístico del modelo núcleo-periferia para poder determinar su pertinencia para el mercado interbancario venezolano.

En el gráfico 1 se muestra el número de bancos estimados en el núcleo (eje de la izquierda) y el error total para cada núcleo normalizado por el número de vínculos y expresado en porcentaje (eje de la derecha).

\section{Gráfico 1. Error/Vínculos}

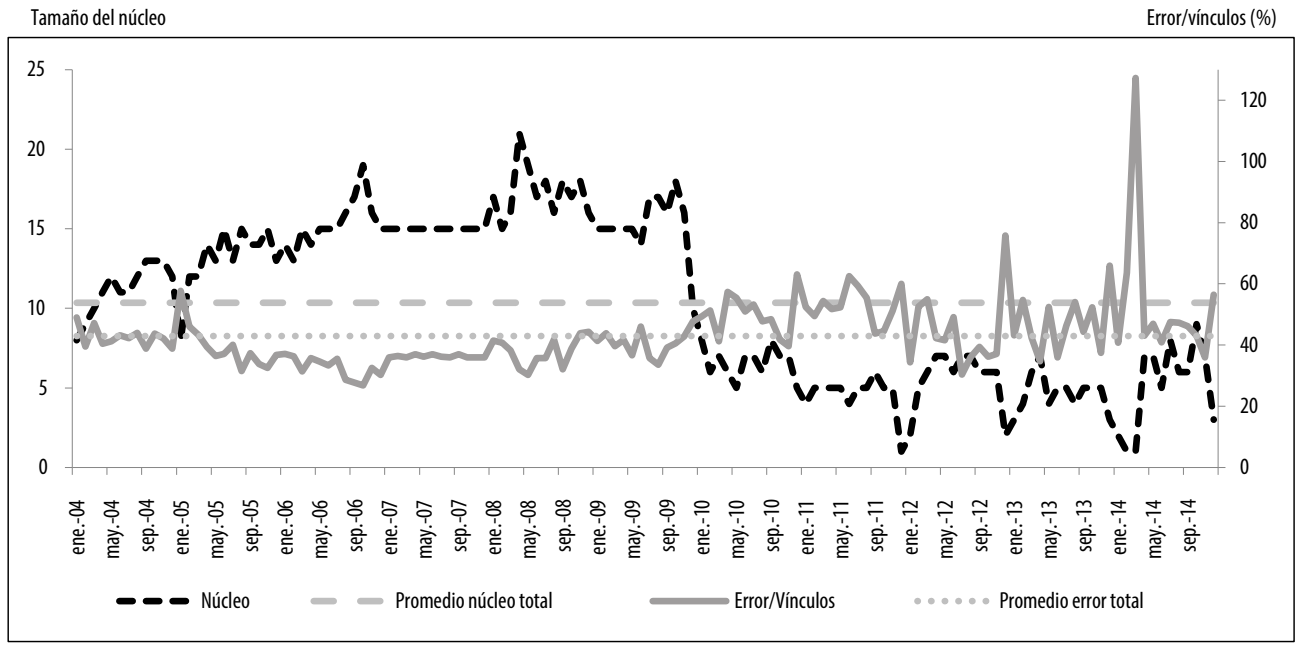

Fuente: elaboración propia

Entre 2004 y 2014, el promedio de los errores fue $43 \%^{10}$. Sin embargo, luego de las fusiones e intervenciones bancarias el error promedio incrementó. Esto ocurrió pese a que luego de 2010 se redujo el número de bancos, el número de vínculos y la densidad de la red, tal como se mostrará más adelante. A continuación mostramos otros indicadores para evaluar mejor el ajuste del modelo.

\subsection{Test de significancia}

En esta sección se evalúa si el mercado interbancario venezolano se aproxima a una estructura jerárquica de dos niveles.

El test inicial para verificarlo es el error del núcleo óptimo, $e\left(C^{*}\right)$, el cual debe ser menor a la unidad. Esta distancia, entre la red y el modelo ideal demuestra

\footnotetext{
10 En el tabla 2 de la sección de resultados, se puede observar que este error promedio fue menor al reportado por Reino Unido y similar al de Italia.
} 
que la estructura en niveles es mejor que un modelo alternativo, donde todos los bancos son periferia. En efecto, el modelo núcleo-periferia admite como posible estructura una red sin vínculos (formada por bancos tipo periferia), pues satisface las condiciones I-IV de la definición; en este caso, el núcleo sería un conjunto vacío. Por lo tanto, al comparar una red sin vínculos con una red observada, se tienen tantos errores como vínculos, así la ecuación [3], se convierte en la ecuación [12]:

$$
e(C)=\frac{\sum_{i} \sum_{j} N_{i j}}{\sum_{i} \sum_{j} N_{i j}}=1
$$

Como el modelo núcleo-periferia optimiza sobre todas las posibles estructuras, no puede tener un error total superior al de una red formada por bancos periferia. En caso contrario, no hay incentivos para ajustar un modelo de dos niveles, ya que se generan más inconsistencias estructurales que un modelo donde todos los bancos son periferia o se comportan como una red aleatoria.

En el gráfico 1 encontramos que entre 2004 y 2014, el error como porcentaje del número de vínculos fue menor a la unidad (o en porcentaje, menor al $100 \%$ ), excepto en marzo de 2014 donde el error fue 127,27 \%. En este mes los datos no reflejan una estructura jerárquica con dos niveles y se corresponden mejor con un modelo alternativo donde todos los bancos son periferia. En el resto de los meses, el modelo núcleo-periferia resultó ser una mejor explicación que un modelo sin estructura.

Ahora bien, en el análisis de Craig y von Peter (2014), el segundo paso para evaluar la aceptación de la estructura en dos niveles consiste en comparar el error $e\left(C^{*}\right)$ con la distribución del error obtenida a partir de redes aleatorias. Consideraremos las redes aleatorias propuestas por Erdös y Rényi (1959) donde la red se obtiene al conectar cualquier par de nodos con una probabilidad fija de independencia $p^{11,12}$. En cada mes se generan 1.000 redes aleatorias de la misma dimensión $(n)$ y densidad $(d)$ que la red interbancaria venezolana con $p=d$. La densidad por definición es la expresión [13]:

$$
d=\frac{\sum_{i=1}^{n} \sum_{j=1}^{n} N_{i j}}{n(n-1)} .100 \%
$$

1 Otras opciones son las redes aleatorias de libre (Barabási y Albert (1999)) y las Small World.

12 Definición: Dado un entero positivo $n$ y un valor de probabilidad $0 \leq p \leq 1$, definimos el gráfico $G(n, p)$ como el gráfico de $n$ vértices, cuyos vínculos se eligen como sigue: para cualquier par de vértices $v, w$, existe un vínculo $(v, w)$ con probabilidad $p$. 
Luego ajustamos el modelo M correspondiente a cada realización. Esto nos permite representar una función de distribución empírica $\mathrm{F}_{e}$ para el error. Definimos la hipótesis,

\section{$\mathrm{H}_{0}$ : $\mathrm{N}$ no muestra una estructura en niveles}

Rechazamos $\mathrm{H}_{o}$ si el test estadístico asociado e(C*) está más cerca de cero que el último percentil de la función de distribución del error encontrado para las redes aleatorias, $\mathrm{F}_{e}(0,01)$, es decir: Rechazamos $\mathrm{H}_{0}$ si: $e\left(\mathrm{C}^{*}\right)<\mathrm{F}_{e}(0,01)$.

El procedimiento anterior se aplica para todo el período en estudio. En el gráfico 2 se encuentra el percentil inferior $\mathrm{F}_{e}(0,01)$ y el $e\left(\mathrm{C}^{*}\right)$ de cada mes para compararlos y evaluar en cuáles casos era posible rechazar o no $\mathrm{H}_{0}$. El error obtenido al ajustar el modelo núcleo-periferia a la red aleatoria de tipo Erdös y Rényi (1959) suele ser superior al obtenido al aplicar el modelo núcleo-periferia a la red observada en el mercado interbancario venezolano. Lo anterior tiene sentido, ya que es poco probable encontrar alguna estructura en una red formada de manera aleatoria13.

Gráfico 2. Error del modelo núcleo periferia en la red observada y $F_{e}(0,01)$ del modelo aleatorio $(\%)$

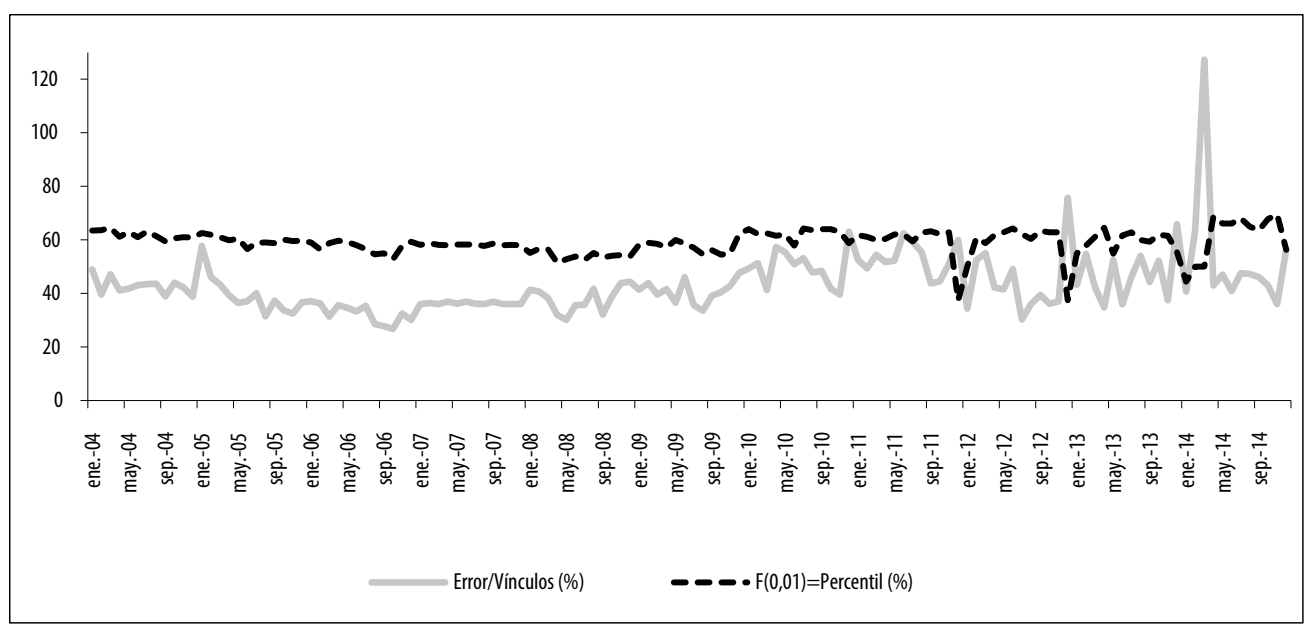

Fuente: elaboración propia

13 También se puede considerar las redes aleatorias de libre escala, las cuales son selectivas por definición, ya que, los gráficos se forman cuando los nuevos nodos se unen a los nodos existentes con una probabilidad proporcional a las últimas conexiones (uniones preferenciales). La probabilidad de que un banco forme vínculos incrementa de forma proporcional con el número de bancos a los que este le presta o de los que recibe recursos. Al aplicar el modelo núcleo-periferia a redes de libre escala con el tamaño y densidad del sistema venezolano en todo el período estudiado, encontramos que, el error total es menor que el obtenido con la red observada, lo cual era de esperarse, ya que, por lo que hemos dicho, las redes de libre escala tienden a mostrar una estructura, y en nuestro caso tenemos la presencia de un componente aleatorio. 
Se encuentra que entre 2004 y 2014 el sistema mostró una estructura jerárquica del tipo núcleo-periferia, excepto en ocho meses, lo que apoya la hipótesis de que en general las conexiones en el sistema no se formaron de manera aleatoria. A partir de diciembre de 2010, es decir, luego de las fusiones e intervenciones se encuentran los ocho meses donde no se rechaza $\mathrm{H}_{o}$ (la línea solida -naranja- supera a la punteada-morada-), a pesar de haber obtenido un error óptimo menor a la unidad.

Antes de las fusiones e intervenciones, es decir, entre enero de 2004 y octubre de 2009 el modelo núcleo-periferia se ajustó bien a la estructura del sistema venezolano. A partir de 2010 se observa un incremento importante de las transacciones entre los propios bancos de la periferia, lo que incrementa el error por encima del promedio, ya que, la partición en la red que genera el núcleo óptimo, es decir, la que satisface la condición mínima de intermediación de las sub matrices CP y PC, genera un incremento de $\mathrm{E}_{22}$. Es decir, hay vínculos en las sub matrices PP, que por definición no deberían existir, lo que sugiere un posible cambio en la estructura del sistema. Por otro lado, $\mathrm{E}_{11}$, es decir, las inconsistencias en la submatriz CC (debido a la falta de vínculos) se han mantenido en torno al promedio, ver gráfico 3.

Gráfico 3. Error como porcentaje del número de vínculos (\%)

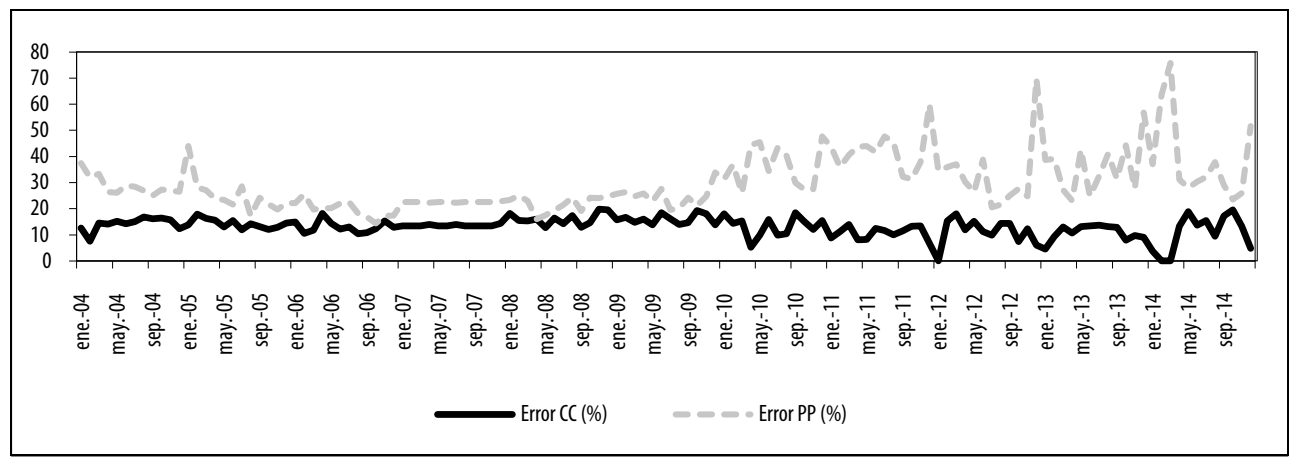

Fuente: elaboración propia

En el gráfico 4, también se tiene que el volumen asociado a las transacciones entre los bancos de la periferia, se incrementa de forma importante al pasar en promedio de $21 \%$ antes del 2010, a 44 \% después del 2011.

Los vínculos y el volumen en las submatrices PP, por lo general, se realizaron en una dirección, es decir, no llegaron a ser relaciones de intermediarios. La revisión de los casos particulares sugiere que algunos bancos que habían estado en el núcleo entre enero de 2004 y 2009 redujeron su actividad interbancaria, y el modelo los reubicó en la periferia, probablemente porque estos bancos se dedican a prestar o a pedir recursos (y no a ambas actividades). A partir de la teoría de redes, una 
supresión aleatoria de bancos o conexiones de la periferia no afecta la estructura del sistema en forma significativa.

Gráfico 4. Proporción del volumen transado en la periferia (con respecto al total)

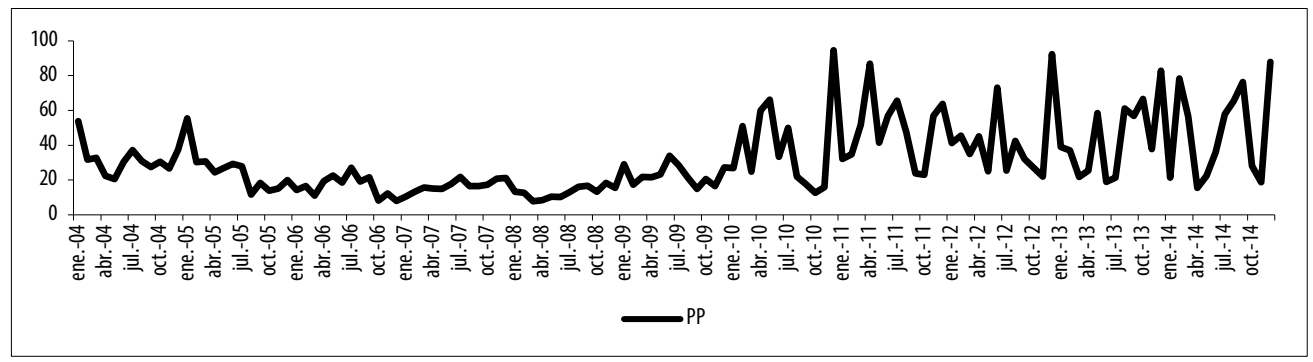

Fuente: elaboración propia

\subsection{Test de robustez}

En esta parte se verifica si los resultados son sensibles a cambios en la red. Dado que el algoritmo de optimización estima el número de bancos en el núcleo, se corrió el algoritmo 1.000 veces en cada mes para ver si la composición del núcleo cambiaba por la re-estimación; sin embargo, se mantienen el tamaño del núcleo y el error total.

Para ver si la estructura en niveles seguía presente se eliminaron algunos bancos de la red (se eliminaron algunas filas y columnas) y se ajustó el modelo núcleo-periferia a la nueva red. Por ejemplo, entre enero 2011 y diciembre 2014, si se trabaja con matrices de dimensión 56x56, sin excluir aquellos bancos que dejaron de tener conexiones luego de las fusiones e intervenciones, los resultados obtenidos se mantienen: el tamaño promedio del núcleo fue de 5 bancos y el error normalizado por el número de vínculos pasó de 48,3 a 48,2 \%, similar a lo que se había encontrado.

\section{ANÁLISIS DE RESULTADOS}

En esta sección se presentan y analizan los resultados obtenidos a partir del modelo núcleo-periferia. Este análisis se basa en la construcción de dos tipos de indicadores: los indicadores de conectividad y los que evalúan los patrones de fondeo tanto por bloques de bancos (núcleo vs periferia), como por bancos individuales.

\subsection{Indicadores de conectividad}

En esta sección se construyen diversos indicadores para medir la conectividad. Algunos de ellos son aplicables a cualquier tipo de red, en tanto que otros están más relacionados con la estructura núcleo-periferia. En total se presentan cinco indicadores para evaluar la conectividad. Todos los indicadores tienden a señalar 
que a partir del 2009, la conectividad se vio reducida y, por tanto, el riesgo asociado a esta.

De manera general, la conectividad total de una red se puede analizar a través de la densidad, es decir, la fracción de vínculos existentes con respecto al número de vínculos posibles (ver gráfico 5). En promedio, entre 2004 y 2014 las redes fueron poco densas, con el 10,48 \% de las relaciones posibles ${ }^{14}$. Este resultado se puede comparar con el de otros estudios en el tabla 2.

\section{Gráfico 5. Densidad del sistema (\%)}

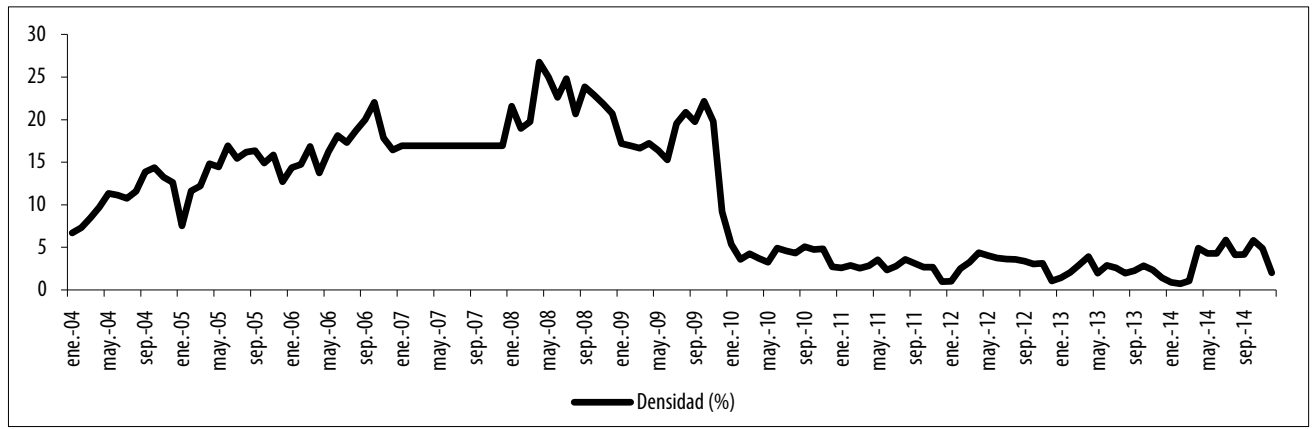

Fuente: elaboración propia

Tabla 2. Comparación con otros trabajos empíricos aplicados a otros países

\begin{tabular}{|l|c|c|c|c|c|c|}
\hline \multicolumn{1}{|c|}{ Variables } & Venezuela & Países bajos & Alemania & Italia & Reino Unido & México \\
\hline N. total de bancos & 56 & 100 & 1.800 & 120 & 176 & 46 \\
\hline Densidad de la red* & $11 \%$ & $8 \%$ & $0,4 \%$ & $15 \%$ & $3,2 \%$ & $26 \%$ \\
\hline $\begin{array}{l}\text { N. promedio de } \\
\text { bancos en el núcleo* }\end{array}$ & 10 & 15 & 45 & 30 & 16 & 16 \\
\hline $\begin{array}{l}\text { Promedio del tamaño } \\
\text { del núcleo* }\end{array}$ & $18 \%$ & $15 \%$ & $2,5 \%$ & $2,5 \%$ & $9,1 \%$ & $35 \%$ \\
\hline $\begin{array}{l}\text { Error como \% de los } \\
\text { vínculos* }\end{array}$ & $43 \%$ & $29 \%$ & $12 \%$ & $42 \%$ & $47 \%$ & $25 \%$ \\
\hline Período de estudio & $\begin{array}{c}\text { Ene04- } \\
\text { Dic14 }\end{array}$ & $\begin{array}{c}1998 \mathrm{Q} \% \\
2008 \mathrm{Q} 4\end{array}$ & $\begin{array}{c}1999 \mathrm{Q} \% 1- \\
2012 \mathrm{Q} 3\end{array}$ & $\begin{array}{c}\text { Ene99- } \\
\text { Dicl0 }\end{array}$ & $\begin{array}{c}\text { II-Semes- } \\
\text { tre } 2011\end{array}$ & $\begin{array}{c}3 \text { Enero al } \\
15 \text { Agosto, } \\
2011\end{array}$ \\
\hline
\end{tabular}

* Corresponde al promedio en el período de estudio

Fuente: adaptación de la tabla resumen de in't Veld y Van Lelyveld (2014)

14 Densidad: es la fracción de vínculos existentes con respecto al número de vínculos posibles. Si tenemos $n$ bancos entonces, el número de vínculos posibles es $n(n-1)$. 
En el tabla 2, puede verse que la red venezolana es en promedio más densa que la encontrada en Alemania, Reino Unido y Los Países Bajos, donde los vínculos efectivos son menores a los posibles. Esto puede deberse a los valores mínimos de transacción que fueron usados en los estudios de estos tres países, para describir las conexiones entre bancos. Esto originó que bancos pequeños con vínculos en el mercado quedaran fuera del análisis. Por otro lado, la red venezolana es más dispersa (menos densa) que la de México e Italia debido a que tiene un mayor número de bancos sin conexión.

Debido a que los bancos del núcleo son en teoría aquellos más conectados, otra forma de medir la conectividad es a partir de la evaluación del tamaño del núcleo.

En el gráfico 6 encontramos el comportamiento del tamaño del núcleo y los intermediarios en el tiempo. En todo el período, el promedio de miembros en el núcleo fue 10 bancos. Los resultados aquí encontrados también confirman que el núcleo es, en efecto, un refinamiento de los intermediarios, quienes en promedio están representados por 31 bancos (el núcleo representa $48 \%$ de los intermediarios para la casi totalidad de los períodos). Sin embargo, es claro que a partir de octubre de 2009, hay un cambio sustancial en el número de bancos que conforman el núcleo. Entre enero de 2004 y octubre de 2009, el promedio de bancos en el núcleo fue de 15. Entre enero de 2011 y diciembre de 2014, el promedio fue de 5 bancos.

Gráfico 6. Número de bancos intermediarios y en el núcleo

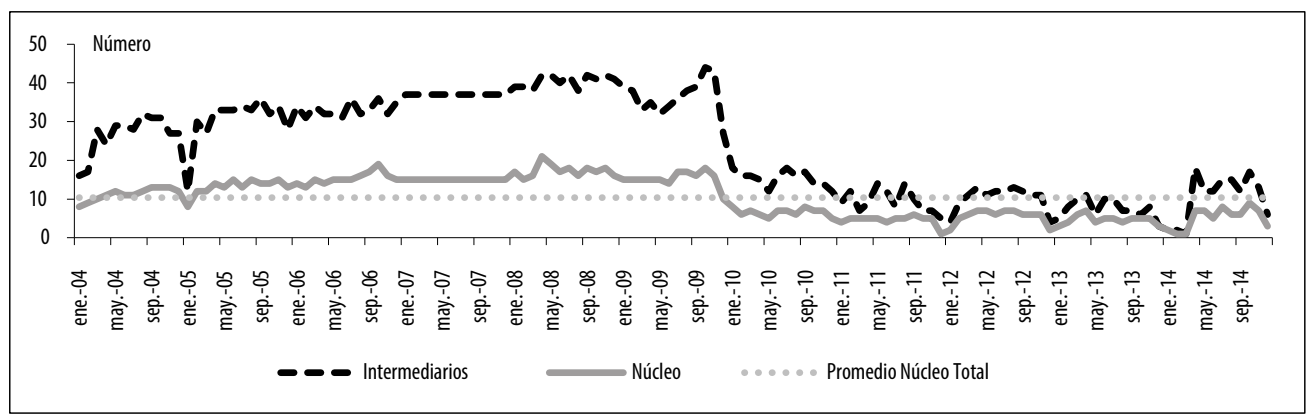

Fuente: elaboración propia

Por otro lado, al comparar el tamaño del núcleo respecto al total de bancos en la red (gráfico 7), encontramos que proporcionalmente, el núcleo también se reduce a partir de 2009, a pesar de que la red interbancaria se redujo de 56 a 33 bancos. Es decir, pareciera que las fusiones e intervenciones llevadas a cabo también condujeran a una reducción de la conectividad del mercado, al disminuir el tamaño relativo del núcleo. 
Gráfico 7. Proporción de bancos en el núcleo (respecto al total de bancos)

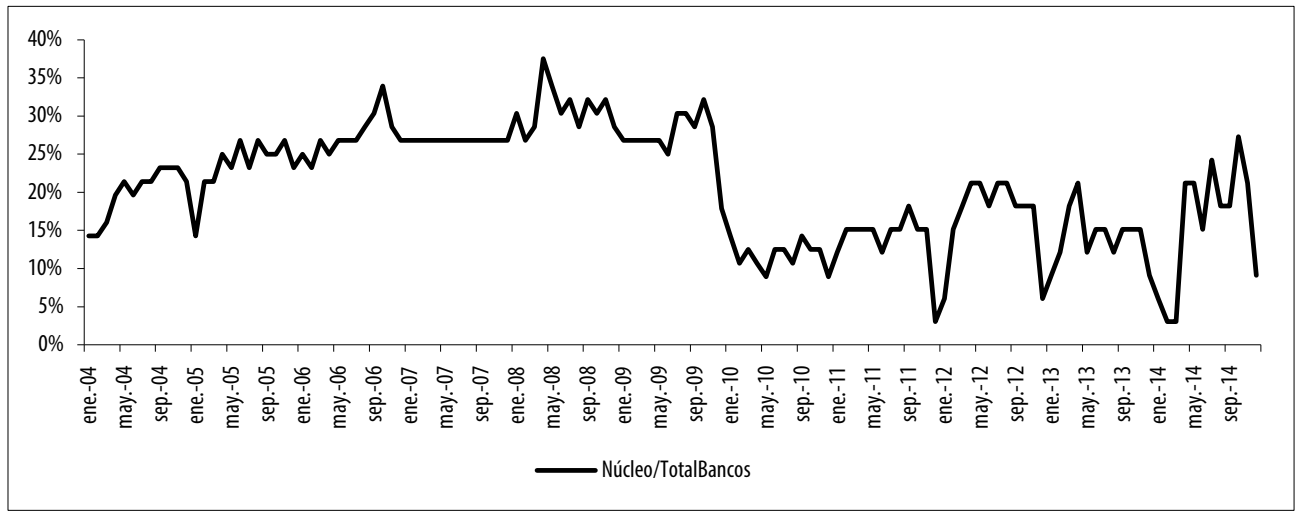

Fuente: elaboración propia

Para tener otra perspectiva sobre la conectividad del núcleo, se analiza el número de conexiones asociadas a las sub-matrices (CC, CP y PC) obtenidas de la partición de la red que realiza el modelo. Primero, calculamos la cantidad de enlaces adyacentes a los bancos del núcleo, normalizado por el número de vínculos existentes en la red (gráfico 8). Es decir, se sumó el número de vínculos que tienen los bancos del núcleo, bien sea, con otros bancos del núcleo (CC) o con bancos de la periferia $(\mathrm{CP}+\mathrm{PC})$, y se comparó al total de vínculos de la red. Se puede observar, que de forma similar en este caso, la conectividad promedio parece reducirse, pero de forma leve, al pasar la proporción de conexiones del núcleo de 76 \%, entre 2004 y 2009, a 62,07 \% entre 2011 y 2014.

Gráfico 8. Proporción de las conexiones del núcleo (respecto al total)

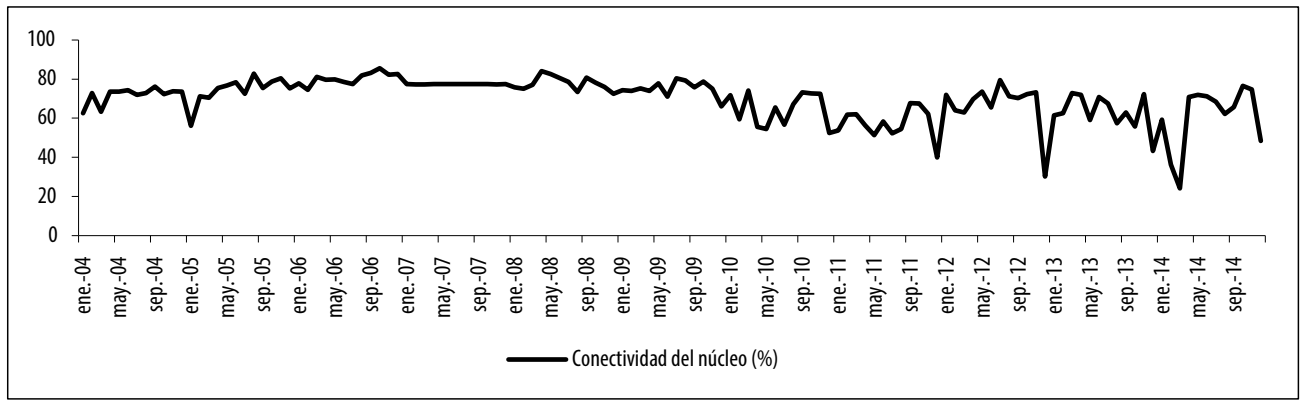

Fuente: elaboración propia

Finalmente, se calculan las conexiones entre los bancos que pertenecen al núcleo (CC) y se compara con el total de vínculos de la red (gráfico 9). También evaluamos 
el volumen asociado a dichos vínculos en relación al total (mensual) de recursos transados en el mercado (gráfico 10). Esto último para corroborar si un número más grande de vínculos implica un mayor movimiento de recursos.

Gráfico 9. Proporción de vínculos dentro del núcleo (respecto al total)

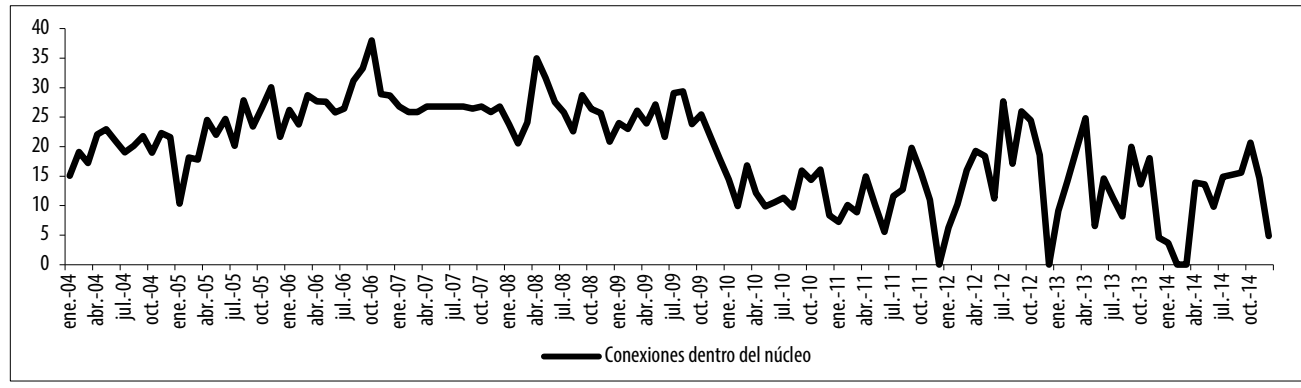

Fuente: elaboración propia

Gráfico 10. Proporción del volumen transado en el núcleo (respecto al total)

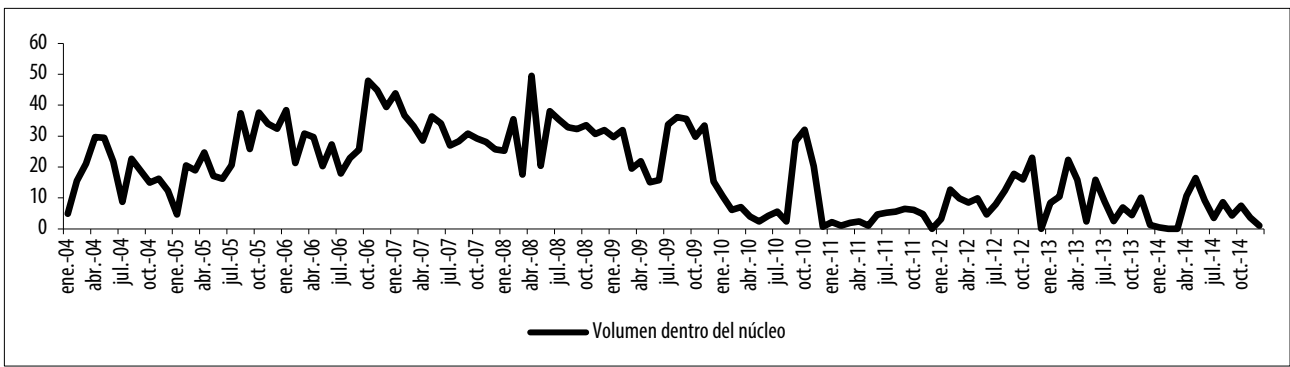

Fuente: elaboración propia

Antes de 2010, las conexiones y el volumen en las submatrices CC tenían un peso importante en el sistema (ver gráficos 9 y 10). Aproximadamente, un $27 \%$ del volumen transado era intercambiado entre bancos del propio núcleo. Luego de las fusiones e intervenciones, los vínculos dentro del núcleo se redujeron y el peso promedio entre 2010 y 2014 de estas transacciones con respecto al volumen total fue $8 \%$ (ver gráfico 10).

Del anterior análisis se desprende que, a raíz de las intervenciones y fusiones iniciadas en 2009, se produjo una reducción de la conectividad en el mercado interbancario que se observa como una clara contracción en el tamaño del núcleo. Esta menor conectividad también ha afectado la relación entre los bancos del sistema, al reducir las transacciones entre los bancos más conectados (los del núcleo). Esto, en general, sugiere que ante cualquier evento de iliquidez el riesgo de propagación tendería a ser menor. 


\subsection{Indicadores de patrones de fondeo por bloques}

Una primera aproximación para entender los patrones de fondeo dentro de la estructura del modelo núcleo-periferia es analizar las conexiones y los volúmenes transados entre los bancos del núcleo y los de la periferia. Para ello calculamos el número de conexiones y volumen en las submatrices (CP y PC). En particular, recordemos que $\mathrm{CP}$ contiene las conexiones que indican préstamos del núcleo a la periferia, en tanto que PC contiene los vínculos que indican préstamos de la periferia al núcleo. Esta información se resume en los gráficos 11 y 12 .

En promedio, entre enero 2004 y diciembre 2014, el 19,8 \% de los vínculos de toda la red corresponde a la matriz CP, mientras que 31,07 \% corresponde a la matriz PC. Es decir, los vínculos de la matriz PC son superiores a los de la matriz $\mathrm{CP}$, lo que nos indica que los vínculos entre el núcleo y la periferia se establecieron en mayor proporción para prestar recursos al núcleo. Esta diferencia entre las dos proporciones se hace más marcada entre enero de 2011 y diciembre de 2014, es decir, luego de las fusiones e intervenciones bancarias (ver gráfico 11).

Gráfico 11. Proporción de vínculos entre el núcleo y la periferia (con respecto al total)

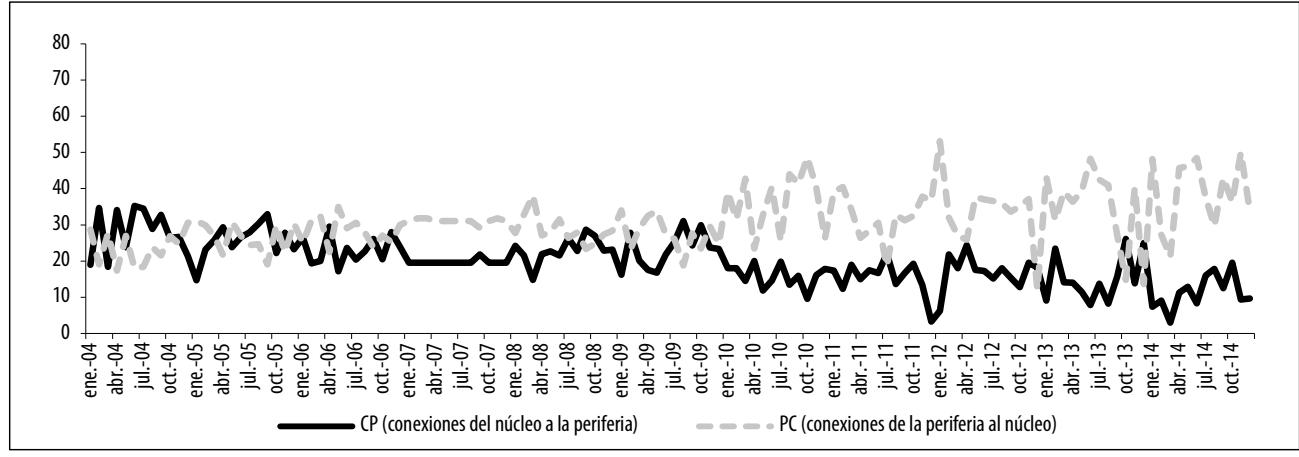

Fuente: elaboración propia

En el gráfico 12 se muestra el volumen asociado a las transacciones (vínculos) del gráfico 11. En promedio, entre enero 2004 y diciembre 2014, el volumen de la matriz CP con respecto al volumen total fue de $14,2 \%$, mientas que el promedio de la matriz PC representa el 35,3\%. Lo anterior también nos indica que durante el período estudiado los bancos del núcleo, en promedio, tendieron a extraer más recursos de la periferia de los que prestaron. El valor de estas transacciones se incrementó a partir de 2010. 
Gráfico 12. Proporción del volumen prestado y recibido por el núcleo en relación a la periferia (con respecto al total)

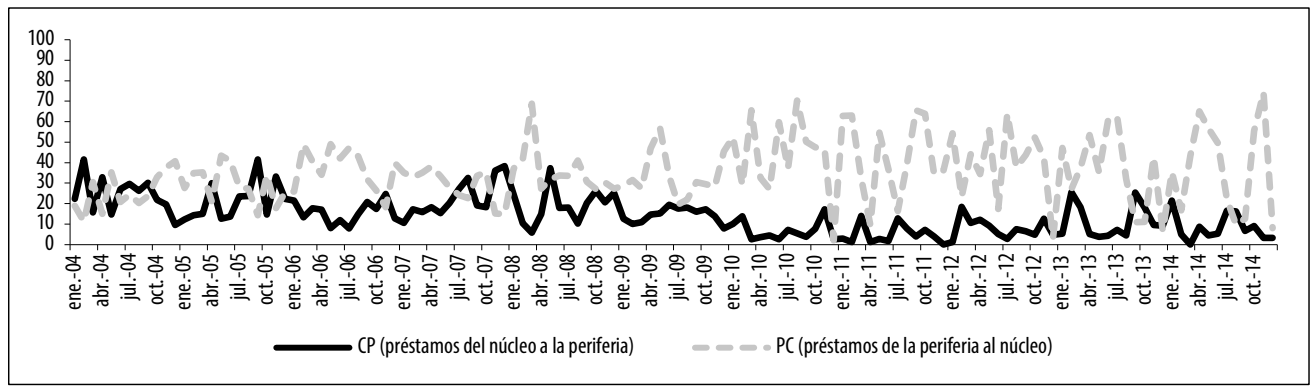

Fuente: elaboración propia

La interpretación de estos indicadores sugiere en general que los bancos del núcleo obtienen recursos de la periferia. Esto se diferencia de otros estudios en los que típicamente el núcleo provee de fondos a la periferia (CP se encuentra por encima de PC) ${ }^{15}$. Estos indicadores también muestran que los hechos de 2009 han afectado los patrones de fondeo entre el núcleo y la periferia, al incrementarse el préstamo de recursos de la periferia al núcleo. Recordemos también que a partir de esta fecha se ha producido una reducción de los bancos y transacciones en el núcleo, y un incremento de los bancos y transacciones de la periferia (Sección 2). Estos dos eventos apuntan a que es probable que bancos que con cierta frecuencia eran antes intermediarios del núcleo, en la actualidad se aboquen a prestar o demandar recursos dentro de la periferia de forma más selectiva, en tanto que los bancos que se mantienen en el núcleo tiendan a hacerlo para obtener mayores fondos de los bancos de la periferia.

Si bien este cambio descrito en la estructura del mercado interbancario se observa de forma clara a partir de las series de fusiones e intervenciones realizadas durante 2009 y 2010, es también posible que otros factores de carácter macroeconómico hayan contribuido a su aparición ${ }^{16}$.

15 Por ejemplo, Fricke y Lux (2012) en su estudio del caso Italiano encuentran que la densidad en CP es muy superior a la densidad del bloque PC.

16 En particular, la ilegalización del mercado cambiario no-oficial por parte del Ejecutivo Nacional a mediados de 2010 y la reducción en la asignación de divisas al sector privado también a partir del 2010, pueden constituir elementos que reducen los niveles de actividad y conectividad del mercado interbancario. Esto es factible en la medida que la redistribución de recursos entre bancos esté motivada por la necesidades de liquidez que implica la liquidación de las divisas asignadas por el Ejecutivo Nacional. 


\subsection{Conectividad y patrones de fondeo por bancos individuales}

El análisis anterior sugiere que los miembros del núcleo (los intermediarios más conectados del sistema) tienden a demandar recursos de la periferia, en especial a partir del 2010. Por tanto, que un banco pertenezca de forma reiterada al núcleo puede ser indicativo de que la ocurrencia de eventos de iliquidez o insolvencia en estos bancos pueda tener repercusiones sistémicas. Es decir, los bancos del núcleo pueden y deben ser sujetos a una mayor supervisión bancaria por parte de las autoridades competentes.

En esta sección, se calculan indicadores por cada banco del sistema, para los dos sub-períodos muestrales, para poder caracterizar su grado de conectividad y patrón de financiamiento. El período 1 va de enero de 2004 a octubre de 2009 (70 meses) y el período 2 va de enero de 2011 a diciembre de 2014 (48 meses). Mientras el grado de conectividad del banco se asocia a su frecuencia de permanencia en el núcleo, su patrón de financiamiento se obtiene calculando la diferencia entre los fondos prestados y los demandados durante dicha permanencia en el núcleo. A continuación, se explica con mayor detalle la construcción de estos indicadores por banco, los cuales no son típicamente calculados en este tipo de literatura ${ }^{17}$. La división de la muestra antes y después de la mayoría de las fusiones e intervenciones bancarias también tiene por objetivo observar con qué frecuencia los bancos que desaparecieron durante este proceso se encontraban en el núcleo o si tenían patrones de fondeo específicos.

El grado de conectividad de un banco $i$ se calcula como el número de períodos en que dicho banco perteneció al núcleo respecto al total de períodos de la submuestra. El patrón de financiamiento del banco $i$ durante los meses que estuvo en el núcleo, se evalúa a través de dos indicadores. El primero se refiere a la diferencia entre el volumen prestado y el volumen recibido en relación con los bancos de la periferia durante ese período sub-muestral. El segundo se refiere a la diferencia entre el volumen prestado y el volumen recibido en relación con los otros bancos del núcleo durante ese mismo período sub-muestral. Para evitar trabajar con valores nominales de los volúmenes, se utiliza la proporción que dichos préstamos (hacia y desde otros bancos) representaron respecto al volumen total de recursos transados en el mes. En particular, el volumen total prestado (otorgado) a la periferia, se denomina $\mathrm{V}_{i}^{\mathrm{OP}}$. De forma similar, el volumen total recibido (demandado) de la periferia, $\mathrm{V}_{i}^{\mathrm{DP}}$. Lo anterior se puede resumir en la expresión [14]. Si esta igualdad es positiva, entonces mientras estuvo en el núcleo, el banco $i$ otorgó préstamos netos a la periferia; si, por el contrario, es negativa, demandó recursos netos de la periferia.

17 La literatura usualmente se concentra en construir y analizar los indicadores por bloques. 


$$
\mathrm{VNP}_{i}=\mathrm{V}_{i}^{\mathrm{OP}}-\mathrm{V}_{i}^{\mathrm{DP}}=\sum_{\left\{t: i \in \mathrm{C}_{t}^{*}\right\}} \sum_{\left\{j: j \in \mathrm{P}_{t}\right\}} \frac{1}{\mathrm{~V}_{t}}\left[\mathrm{~V}_{i j t}-\mathrm{V}_{j i t}\right]
$$

Donde, $\mathrm{VNP}_{i}$ es el volumen (relativo) neto de recursos prestados por el banco $i$ a la periferia durante los meses que estuvo en el núcleo.

De igual manera en la expresión [15], $\mathrm{VNC}_{i}$ es el volumen (relativo) neto de recursos prestados por el banco $i$ a los otros bancos del núcleo durante los meses que estuvo en el núcleo:

$$
\mathrm{VNC}_{i}=\mathrm{V}_{i}^{\mathrm{OC}}-\mathrm{V}_{i}^{\mathrm{DC}}=\sum_{\left\{t: i \in \mathrm{C}_{i}^{*}\right\}\left\{\left\{j \neq i: j \in \mathrm{C}_{i}^{*}\right\}\right.} \frac{1}{\mathrm{~V}_{t}}\left[\mathrm{~V}_{i j t}-\mathrm{V}_{j i t}\right]
$$

El gráfico 13 resume para cada banco su relación con la periferia y con los otros miembros del núcleo, entre enero de 2004 y octubre de $2009 .{ }^{18}$ En este gráfico, se identifican con un color diferenciado (barras verdes con marcas puntos) aquellos bancos que luego fueron intervenidos y fusionados. El gráfico 14 resume las relaciones por banco entre enero de 2011 y diciembre de 2014. En cada gráfico los bancos se ordenaron de forma creciente de acuerdo con el número de veces que aparecieron en el núcleo.

Una apreciación general que se deriva de los gráficos 13 y 14 es que los bancos de mayor tamaño tienden a estar muy pocas veces en el núcleo ${ }^{19}$. Por tanto, estos bancos presentan indicadores VNP y VNC muy cercanos a cero, lo que revela que durante su estadía en el núcleo no fueron ni prestamistas ni prestatarios netos de fondos. Por el contrario, los bancos con mayor presencia en el núcleo y demandantes netos de recursos tendieron a ser bancos de menor tamaño. Una potencial explicación a este fenómeno es la vigencia del control cambiario. Con su implementación en 2003, los niveles de liquidez del sistema se incrementaron sustancialmente debido a la reducción de las operaciones cambiarias. En este contexto los niveles de actividad en el mercado interbancario también se redujeron, lo que también pudo haber reducido las conexiones entre bancos. Por tanto, los bancos de mayor tamaño se especializaron en prestar fondos sin intermediar entre ellos, siendo generalmente catalogados como bancos de la periferia. Por el contrario, los bancos más conectados terminaron siendo aquellos cuyas necesidades de liquidez fueron mayores.

18 Antes de hacer este análisis, en el primer período ordenamos los bancos por orden alfabético y luego los identificamos con las etiquetas B1, B2,..., B56. En el segundo período con 33 bancos, eliminamos las instituciones que desaparecieron y mantuvimos las etiquetas asignadas a cada banco en el primer período de estudio con 56 bancos.

19 A pesar de que no se muestra ningún indicador de tamaño, sabemos que de la totalidad de nuestro sistema bancario, los cuatro bancos que mantienen más del 74\% de los depósitos del público tuvieron poca presencia en el núcleo durante el período analizado. Más aún, luego de las fusiones y las intervenciones bancarias estos cuatro bancos estuvieron menos del 10\% de las veces en el núcleo. 
Gráfico 13. Período de estudio 1, enero 2004 a octubre 2009

a) $V N P_{i}$ : indicador del volumen neto prestado a la periferia por banco

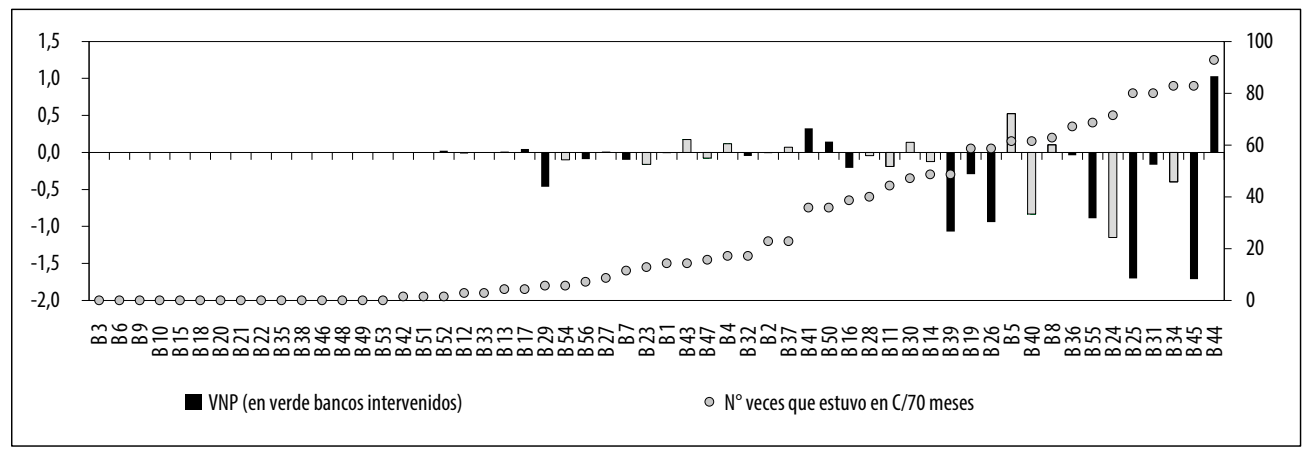

b) $V N C_{i}$ : indicador del volumen neto prestado al núcleo por banco

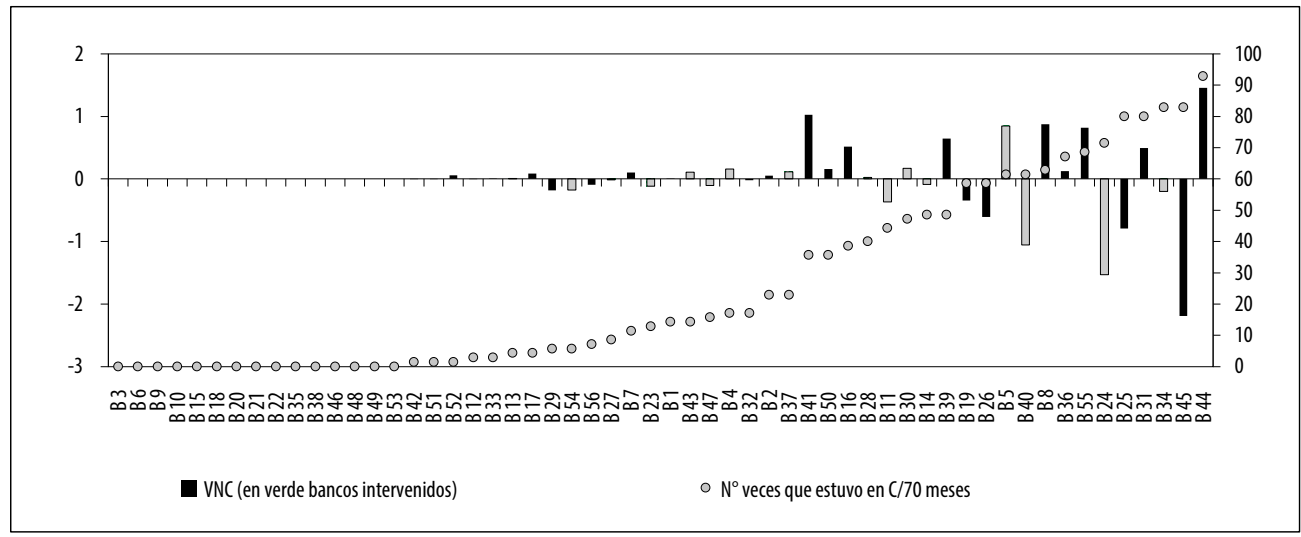

Fuente: elaboración propia

En los gráficos 13 a) y 14 a) encontramos que, en general, el indicador VNP tiende a ser más negativo en los bancos que estuvieron con más frecuencia en el núcleo. En particular, para el segundo período el indicador VNP tiende a tener valores más negativos que en el primer período, a pesar de considerar un número de meses menor. Esta observación podría indicar que los bancos que se mantienen en el núcleo después de las fusiones e intervenciones profundizan su fondeo con bancos de la periferia.

Los gráficos 13 b) y 14 b), en general reflejan que estos bancos con mayor permanencia en el núcleo no necesariamente intermediaron los recursos obtenidos de la periferia hacia los otros bancos del núcleo. Esto se deduce cuando VNP $<0$ y 
VNC $\leq 0$ En estos casos, la obtención neta de recursos de la periferia (y/o del propio núcleo) tiene como objetivo satisfacer necesidades de liquidez ${ }^{20}$.

Gráfico 14. Período de estudio 2, enero 2011 a diciembre 2014

a) $V N P_{i}:$ : indicador del volumen neto prestado a la periferia por banco

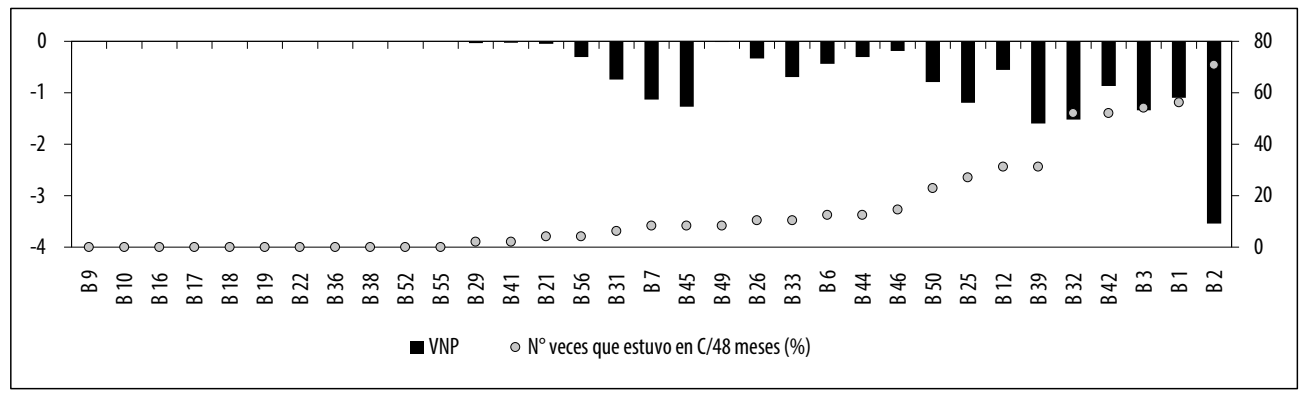

b) $V N C_{i}:$ : indicador del volumen neto prestado al núcleo por banco

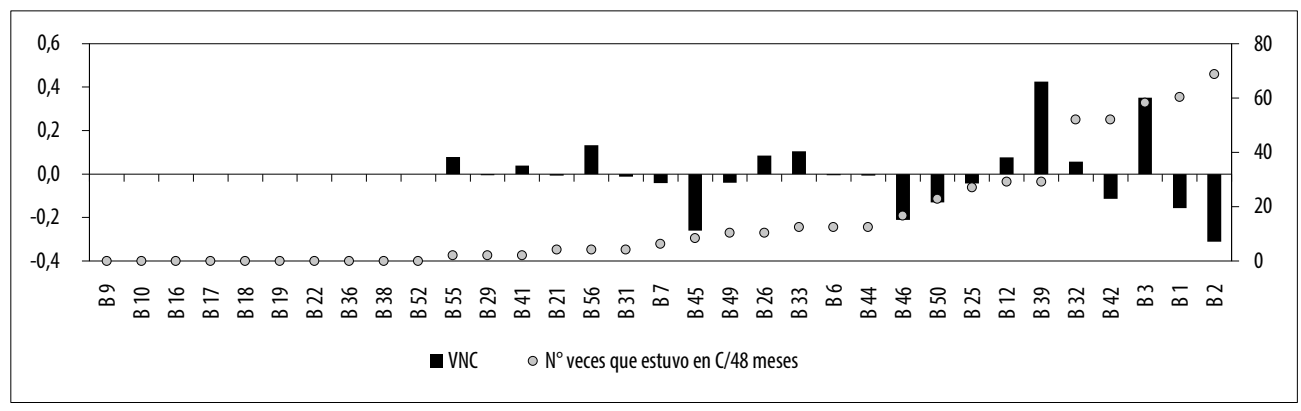

Fuente: elaboración propia

Al analizar en el gráfico 13 los bancos que posteriormente fueron intervenidos o fusionados (barras en verde), se observa que las acciones de la superintendencia afectaron una variedad amplia de instituciones financieras. Esto en parte es consistente con la diversidad de objetivos que se suelen asociar a dichas intervenciones. Sin embargo, surge como un hecho interesante que, si un banco estuvo en el núcleo en la primera parte de la muestra más de $40 \%$ de las veces, la probabilidad de haber sido intervenido o fusionado fue de $45 \%$. En otras palabras, casi la mitad de los bancos que fueron intervenidos y fusionados estuvieron presentes en el núcleo más del 40 \% de las veces entre 2004 y 2009 (algunos incluso, estuvieron más del 70 \% de las veces). Para el período más reciente, se puede afirmar que todos aquellos

20 Se podría inferir que aquéllos bancos en los que VNP tiende a ser similar en magnitud, pero de signo contrario a VNC, la motivación para recibir recursos netos del núcleo o la periferia es el arbitraje de recursos entre grupos de bancos. 
bancos con una permanencia en el núcleo mayor a 50 \% muestran de forma clara necesidades persistentes de liquidez.

\section{CONCLUSIONES}

El modelo núcleo-periferia hasta ahora se ha utilizado mayoritariamente para caracterizar la estructura de los mercados interbancarios en forma jerárquica. En los estudios empíricos aplicados a otros países, los bancos del núcleo tienden a ser los bancos más grandes del sistema, los cuales, en muchos casos, participan como mayoristas del mercado monetario (market makers).

En esta aplicación al mercado interbancario venezolano hemos mostrado cómo la información que se deriva del modelo puede ser utilizada para entender dimensiones del desempeño bancario que se relacionan con la aparición del riesgo sistémico. Estas dimensiones se refieren a la conectividad del sistema y a la detección de patrones de fondeo por grupos de bancos y bancos individuales que puedan indicar la existencia de problemas de liquidez. En particular, a lo largo del trabajo se ha mostrado cómo interpretar algunos de los indicadores comúnmente usados en la literatura y otros indicadores por banco no explorados en otros trabajos.

La aplicación de esta herramienta al caso venezolano es de particular interés porque durante el período seleccionado se encuentra en vigencia un control cambiario que, además, coincide con una serie de fusiones e intervenciones bancarias, concentradas en un lapso aproximado de un año, que dividen la muestra en un antes y un después. Los resultados discutidos muestran que estos dos elementos de política cambiaria y financiera son los que potencialmente explican la particularidad de la estructura del mercado interbancario y de los cambios que se generan en el tiempo. Por un lado, la vigencia del control cambiario posiblemente redujo la intermediación interbancaria y permitió la prevalencia de aquellas relaciones basadas en requerimientos de liquidez. De allí, que los bancos del núcleo tiendan a especializarse en la recepción de recursos de la periferia. Por otro lado, el episodio de ajuste financiero generó cambios importantes en la estructura del mercado que se pueden resumir en dos ideas principales.

La primera es que, con la intervención y fusión de los bancos iniciada en 2009, la conectividad del mercado parece haberse reducido de forma sustancial. Esto se deduce de la disminución del tamaño del núcleo y en general, de la conectividad de los bancos del núcleo con el resto de los bancos. Esta menor conectividad ocurre en asociación a un contexto macroeconómico en el que se ilegaliza el mercado cambiario no-oficial y las asignaciones de divisas al sector privado comienzan a 
mermar. En términos generales, esta menor conectividad podría hacer pensar en una disminución del riesgo sistémico, en tanto la ocurrencia de choques idiosincráticos a bancos tendría una menor probabilidad de ser transmitida al resto de los bancos.

En contraposición, la segunda conclusión que se extrae de los resultados señala que, posterior a los ajustes financieros, los patrones de fondeo en el mercado interbancario parecen haberse modificado. En particular, los bancos del núcleo redujeron sus vínculos y volúmenes transados con el resto de los bancos del núcleo, pero, a su vez, incrementaron sus vínculos y volúmenes transados con los bancos de la periferia. Sin embargo, estas mayores conexiones del núcleo en relación con la periferia ocurren principalmente para obtener recursos de ésta. Cuando se observan indicadores similares calculados por banco, se confirma que a partir de 2011, los bancos que se mantienen más veces presentes en el núcleo tienden a demandar mayores préstamos netos a los bancos de la periferia. Esta última observación pareciera sugerir que pese a la reducción del núcleo, los bancos que se mantienen en él, tienden a tener necesidades persistentes de liquidez que incrementan el riesgo sistémico.

En términos amplios, este trabajo muestra que la aplicación del modelo núcleo-periferia puede servir para la detección de potenciales problemas de orden sistémico, y para indicar sobre qué bancos debe focalizarse la supervisión. Sin embargo, mayores ganancias asociadas a la aplicación de este modelo podrían obtenerse a partir de la modelación y simulación de la trasmisión del riesgo al usar esta estructura. Es por ello, que este trabajo deja una agenda de investigación abierta que se concentrará en el desarrollo de mayores herramientas útiles para la supervisión bancaria y aplicación de normas macro-prudenciales.

\section{BIBLIOGRAFÍA}

Aldasoro, Iñaki y Alves, Ivan (2015). Multiplex interbank networks and systemic importance: an application to European data. En: SAFE Working paper, No. 102, 43p.

Allen, Franklin y Gale, Douglas (2000). Financial Contagion. En: Journal of Political Economy, Vol. 108, No. 1, p. 1-33

Anand, Kartik; Craig, Ben y von Peter, Goetz (2014). Filling in the Blanks: Network Structure and Interbank Contagion. En: Working Paper of the Federal Reserve Bank of Cleveland, No 14-16, p. 1-15

Barabási, Albert-László (2014). Network Science. Cambridge University Press; 1 edition (August), $474 \mathrm{p}$.

Barabási, Albert-László y Albert, Réka (1999). Emergence of scaling in random networks. En: Science, Vol. 286, No. 5439, p. 509-512. 
Bastos e Santos, Edson y Cont, Rama (2010). The Brazilian Interbank Network Structure and Systemic Risk. En: The Banco Central do Brasil Working Paper Series 2019, 54p.

Borgatti, Stephen y Everett, Martin (1999). Models of core/periphery structures. En: Social Networks, Vol. 21, No. 4, p. 375-395.

Boss, Michael; Helmut Elsinger, Martin Summer y Stefan, Thurner (2004). Network Topology of Interbank Market. En: Quantitative Finance, Vol. 4, No. 6, p. 677-684

Cáribas, Alejandro (2011). ¿Mini crisis financiera 2009, 2010? En: Ediciones Paredes II, C.A. Caracas-Venezuela, 156p.

Craig, Ben y Von Peter, Goetz (2014). Interbank Tiering and Money Center Banks. En: Journal of Financial Intermediation, Vol. 23, No. 3, p. 322-347

Craig, Ben; Fecht, Falko y Tümer-Alkan, Günseli (2015). The role of interbank relationships and liquidity needs. En: Journal of Banking \& Finance, Vol. 53, p. 99-111

Doreian, Patrick; Batagelj, Vladimir y Ferligoj, Anuška (2005). Generalized Block-modelling. Cambridge University Press, 384p.

Degryse, Hans y Nguyen, Grégory (2007). Interbank exposures: An empirical examination of contagion risk in the belgian banking system. En: International Journal of Central Banking, Vol. 3, No. 2, p. 123-171.

Erdös, Paul y Rényi, Alfréd (1959). On random graphs I. En: Publicationes Mathematicae, Vol. 6, p. 290-297.

Frait, Jan y Komárková, Zlatuše (2011). Financial stability, systemic risk and macroprudential policy. En: Financial Stability Report 2010/2011, Czech National Bank, p. 96-111.

Fricke, Daniel y Lux, Thomas (2012). Core-Periphery Structure in the Overnight Money Market: Evidence from the e-MID Trading Platform. En: Kiel Working Paper No.1759, 60p.

FSB, IMF, BIS (2009). Guidance to Assess the Systemic Importance of Financial Institutions, Markets and Instruments: Initial Considerations. En: Financial Stability Board, International Monetary Fund and Bank for International Settlements. Report to G20 Finance Ministers and Governors, October 2009, 27p.

Furfine, Craig (2003). Interbank Exposures: Quantifying the Risk of Contagion. En: Journal of Money, Credit, and Banking, Vol. 35, No. 1, p. 111-128.

in't Veld, Daan y van Lelyveld, Iman (2014). Finding the core: Network structure in interbank markets. En: Journal of Banking \& Finance, Vol. 49, No. 3, p. 27-40.

Hojman, Daniel y Szeidl, Adam (2008). Core and Periphery in Networks. En: Journal of Economic Theory, Vol. 139, No. 1, p. 295-309.

Komárková, Zlatuše; Hausenblas, Václav y Frait, J. (2012). How to identify systemically important financial institutions. En: Financial Stability Report 2011/2012, Czech National Bank, p. 100-111.

Langfield, Sam; Liu, Zijun. y Ota, Tomohiro (2014). Mapping the UK interbank system. En: Bank of England. Working paper No. 516, 38p. 
León, Carlos; Lía, Clara; Cepeda, Freddy y Sarmiento, Miguel (2012). La importancia de la conectividad para identificar y medir fuentes de riesgo sistémico. En: Banco de la República. Reportes del emisor. Investigación e información económica, No. 161, 5p.

León, Carlos; Pérez, Jhonatan y Mariño, Ricardo (2015). Aproximación a la estructura del mercado cambiario colombiano desde el análisis de redes. En: Banco Central de la República de Colombia. Borradores de Economía. No. 867, 26p.

Liu, Zijun y Quiet, Stephanie (2015). Banking sector interconnectedness: what is it, how can we measure it and why does it matter?. En: Bank of England Quarterly Bulletin, Vol. 55, No 2, 9p.

Minoiu, Camelia (2012). Atrapados en la red. En: Finanzas \& Desarrollo, Septiembre 2012, Fondo Monetario Internacional, Vol. 49, No. 3, p. 15-17.

Pagliacci, Carolina (2008). El Mercado venezolano de fondos overnight: un Mercado de órdenes limitadas con restricciones al crédito. En: Banco Central de Venezuela. Colección Economía y Finanzas. Serie Documentos de Trabajo, No. 96, 18p.

Solis-Montes, Mahetabel Pável (2013). The structure of the Mexican Interbnak Market. En: MIMEO. Banco de México.

Soramäki, Kimmo; Bech, Morten; Arnold, Jeffrey; Glass, Robert y Beyeler, Walter (2007). The topology of interbank payment flows. En: Physica A: Statistical Mechanics and applications, Vol. 379, No. 1, p. 317-333.

Tirole, Jean (2011). Illiquidity and all its friends. En: Journal of Economic Literature, Vol. 49, No. 2, p. 287-325.

Upper, Christian (2011). Simulation methods to assess the danger of contagion in interbank markets. En: Journal of Financial Stability, Vol. 7, No. 3, p. 111-125.

Upper, Christian y Worms, Andreas (2004). Estimating bilateral exposures in the German interbank market: is there a danger of contagion?. En: European Economic Review, Vol. 48, No. 4, p. 827-849

Yellen, Janet (2013). Vice Chair Board of Governors of the Federal Reserve System at the American Economic Association/American Finance Association Joint Luncheon. San Diego, California, 4 de enero de 2013. 